\title{
Ontology Change Management and Identification of Change Patterns
}

\author{
Muhammad Javed • Yalemisew M. Abgaz • \\ Claus Pahl
}

Received: 16 April 2012 / Revised: 21 November 2012 / Accepted: 20 December 2012 / Published online: 22 May 2013

(C) Springer-Verlag Berlin Heidelberg 2013

\begin{abstract}
Ontologies can support a variety of purposes, ranging from capturing the conceptual knowledge to the organisation of digital content and information. However, information systems are always subject to change and ontology change management can pose challenges. In this sense, the application and representation of ontology changes in terms of higher-level change operations can describe more meaningful semantics behind the applied change. In this paper, we propose a four-phase process that covers the operationalization, representation and detection of higher-level changes in ontology evolution life cycle. We present different levels of change operators based on the granularity and domain-specificity of changes. The first layer is based on generic atomic level change operators, whereas the next two layers are user-defined (generic/domain-specific) change patterns. We introduce layered change logs for the explicit operational representation of ontology changes. We formalised the change log using a graph-based approach. We introduce a technique to identify composite changes that not only assists in formulating ontology change log data in a more concise manner, but also helps in realizing the semantics and intent behind any applied change. Furthermore, we identify frequent change sequences that are applied as a reference to discover reusable, often domain-specific and usage-driven change patterns. We describe the pattern identification algorithms and evaluate their performance.
\end{abstract}

M. Javed (凶) · Y. M. Abgaz · C. Pahl

Centre for Next Generation Localisation (CNGL),

School of Computing, Dublin City University, Dublin 09, Ireland

e-mail: mjaved@computing.dcu.ie

Y. M. Abgaz

e-mail: yabgaz@computing.dcu.ie

C. Pahl

e-mail: claus.pahl@dcu.ie
Keywords Customizable ontology evolution · Ontology change patterns · Pattern-based ontology evolution · Change log graph · Graph-based composite change detection . Change pattern discovery algorithms

\section{Introduction}

Ontologies become essential for knowledge sharing activities, especially in areas such as bio-informatics, semantic web, educational technology systems, indexing and retrieval, etc. Ontology-based content models help to take a step forward from traditional content management systems (CMS) to conceptual knowledge modelling, to meet the requirements of the semantically aware content-based systems (CBS). While some generic ontologies (like upper ontologies) evolve at a slower pace, we have been working with non-public ontologies [formalised using the Web Ontology Language (OWL)] used to annotate content in large-scale information systems. In this context, changes happen on a daily basis, triggered by changes in software, its technical or domain environment. Systematic change becomes here a necessity to enable controlled, accountable and predictable ontology evolution.

Ontology evolution is defined in different ways $[13,16$, 45]. A comprehensive definition is given as "the timely adaptation of an ontology to changed business requirements, to trends in ontology instances and patterns of usage of the ontology based application, as well as the consistent management/propagation of these changes to dependent elements" [45]. The change operators are the building blocks of ontology evolution. Different layers of change operators have been suggested in past $[27,34,44]$. However, the identified change operators focus on generic and structural changes lacking domain-specificity and abstraction. These solutions 
lack adequate support for different levels of granularity at different levels of abstraction. Furthermore, for semantically enhanced information systems, a coherent representation of such ontology changes conveying the semantics of changes is essential.

In this paper, we present a four-phase ontology change management system that covers the operationalisation and the identification of higher-level ontology change patterns (Fig. 1). These phases include change operationalisation, change representation, change semantic capturing and change pattern discovery. Few sections of the presented ontology change management system have already been presented in our previously published papers. The explicit distinction between already published and the new work is given below in each section of the phases.

Phase 1: change operationalisation We present a layered change operator framework (discussed in Sect. 4) which consists of three different levels of change operators, based on granularity and domain-specificity. These layered change operations capture the real changes in the selected domains. The first two layers are generic change operators that can be applied on any domain. The changes at a higher level of granularity, which are frequent in a domain and are often neglected by the lower-level compositional change operators addressed in the literature, are captured as domain-specific change operators at level three. The layered change operator framework has been introduced in [22]. However, an underlying structural model has been added to complement the behaviour model.

Phase 2: change representation The implementation of the change operator framework is supported through layered change logs (discussed in Sect. 5). Representing ontology changes as higher-level change operations describes the semantics behind any of the applied change operation. Using higher-level representation of ontology changes, the intent of the applied changes can be explicitly expressed. While the layered change log and a graph-based foundation has been suggested in [24], we substantially expand this here. We exploit the ontology change logs and the pattern recognition techniques to identify the ontology change patterns. To do so, we formalised the ontology change logs using a graph-based approach (discussed in Sect. 5.3).

Phase 3: change semantic capturing The atomic change operations can only represent the addition or deletion of any particular knowledge in the ontology. We utilized the graphbased representation of ontology changes to identify the composite change patterns (discussed in Sect. 6), which cannot be captured by simple queries on ontology change logs. The composite change operations provide more semantic information of how an ontology changed as well as specific reasons and consequences of operations at a higher level.

Phase 4: change pattern discovery: The discovery of domain-specific change patterns (discussed in Sect. 7) pro-

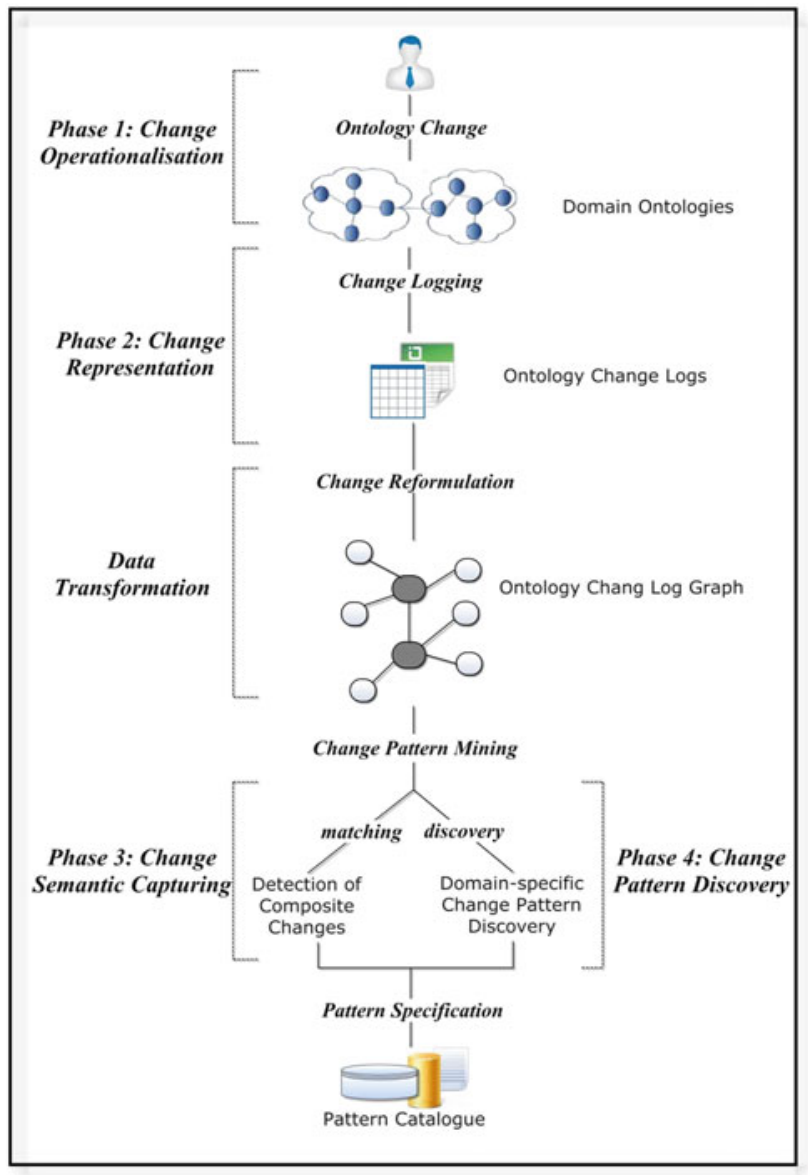

Fig. 1 Proposed four-phase ontology change management

vides an opportunity to define reusable change patterns that can be implemented in existing knowledge management systems. One of the key benefits of change pattern discovery approach is its integration with an ontology editing framework for pattern-driven ontology evolution. While the graphbased patterns discovery approach along with the algorithms has been presented [23], we provide a detailed evaluation here.

The paper is structured as follows. Related work is discussed in Sect. 2. In Sect. 3, we talk about ontology change management in general. Sections 4-7 give detailed description of each phase of the proposed ontology change management system. Experimental results and an evaluation are discussed in Sect. 8 and we end with some conclusion in Sect. 9.

\section{Related Work}

The dynamic nature of knowledge in every conceptual domain requires ontologies to change over time. The reason for change in knowledge can be changes in the domain, the specification, the conceptualization or any combination 
of them [33]. Some changes are about the introduction of new concepts, removal of outdated concepts and changes in the structures and the description of concepts. A change in an ontology may originate from a domain knowledge expert, a user of the ontology or a change in the application area [32].

Based on the different perspectives of the researchers, there are different solutions provided to handle ontology evolution $[4,7,16,17,28,29,32,39,41,44,50]$. The author in [44] discusses the complexity of the ontology change management process and presents a six phase ontology evolution process. She discusses the representation of generic changes and categorized them into elementary, composite and complex. In contrast to our work, aspects such as granularity, domain-specificity and abstraction are not included. In [7], the authors present a pattern-driven ontology evolution approach with defined participants and execution steps. In [41], the authors present a pattern-driven approach for the evolution of RDF knowledge bases. The approach is based on a declarative definition of evolution patterns.

In [33], the authors provide a set of possible ontology change operations based on the effect with respect to the protection of the instance-data availability. Their aim is ensuring the validity of the instance level data rather than the schema level or domain-specific operations. In [40], the impact of ontology change to the validity of the instance availability is discussed and changes are subdivided into two categories, i.e. structural and semantic changes. Though their work addresses semantic changes, our work takes the semantic changes further and proposes domain-specific change patterns for semantic changes. In [46], the authors present a declarative approach to represent the semantics of changes, considered as a reconfiguration-design problem. Their work is focused on the realization of the changes, whereas our work is focused on identifying domain-specific change patterns.

Representation of ontology changes using higher-level change operations was first proposed by Stojanovic [45] and Klein [27]. Recently, some researchers have focused on representation and detection of higher-level ontology changes $[14,35]$. In [35], the author proposed a language that allows formulating the intuition behind any applied change in an RDF graph and provided the change detection algorithm with respect to the proposed language. To detect composite changes, an algorithm compares two versions of the RDF graph (given in the form of triples in RDF/S). The algorithm first picks up a triple added to (or deleted from) the previous version of the knowledge base and looks for potential changes in a look-up table. Based on the potential changes identified, the algorithm searches for other added (or deleted) triples to detect certain type of ontology change. In contrast to their approach, we identify the composite changes from a single ontology change log graph and do not use different versions of an ontology for comparison. Thus, in order to realize that a certain ontology element exists in the previous version of the ontology, we search for an inclusion change operation (that adds the element in the ontology) in all previous change log sessions. If we find one, there must not exist a (later) exclusion change operation that cancels out the previous inclusion change until the start of the current session is reached.

Evolutionary strategies were first proposed by Stojanovic [44] where she considered them as solutions for keeping the ontology consistent at each resolution point. The resolution point refers to the places in ontology evolution where the user may adopt more than one option to keep the ontology consistent. For example, the instances of a deleted concept $x$ will be (1) deleted, (2) the unique instance will be deleted or (3) are linked to the parent concepts of $x$.

A lot of research has been done on mining of process models from event $\log$ data $[1,5,8,36,47,48]$. An early work that relied on the activity logs for producing formal process models corresponding to actual process execution is given in [5]. Metrics such as event frequency and regularity were taken into consideration to discover process models. Results show its usefulness in activities such as process model discovery, re-engineering, software process improvement, etc. In [48], the focus is on detection of invisible tasks from event logs. Their definition of invisible tasks is tasks that exist in a process model, but not in its event log (such as initialize, skip, switch, redo, etc.). In contrast to their work, we are interested in the detection of composite changes such as split, move, merge, etc. In [36], the author defined the Event and Process Mining ontologies. These two ontologies can be used to incorporate semantics in the log, related to the event types and the process instances.

The mining of sequential patterns was first proposed by Agrawal and Srikant [2]. Since then, many sequential pattern mining algorithms, often based on specific domains $[3,31$, $37,43,51]$, have been suggested. In the domain of DNA or protein sequences, BLAST [3] is one of the most well-known algorithms. Given a query sequence (candidate sequence), it searches for a match from the databases. In contrast, we focus on mining of change sequences (patterns) from an ontology change database. In [51], the author proposed the MCPaS algorithm to answer the problems of mining complex patterns with gap requirements. Similar to our approach, it allows pattern generation and growing to be conducted step by step using gap-constrained pattern search.

Several algorithms focus on graph-based pattern discovery $[20,21,30,49]$. In [21], the author proposes an aprioribased algorithm, called AGM, to discover frequent substructures. In [49], the authors propose the gSpan (graph-based Substructure pattern mining) algorithm for mining frequent closed graphs and adopted a depth-first search strategy. In contrast to our work, their focus is on discovering frequent graph substructures without candidate sequence generation. A chemical compound dataset is compared with results of the 


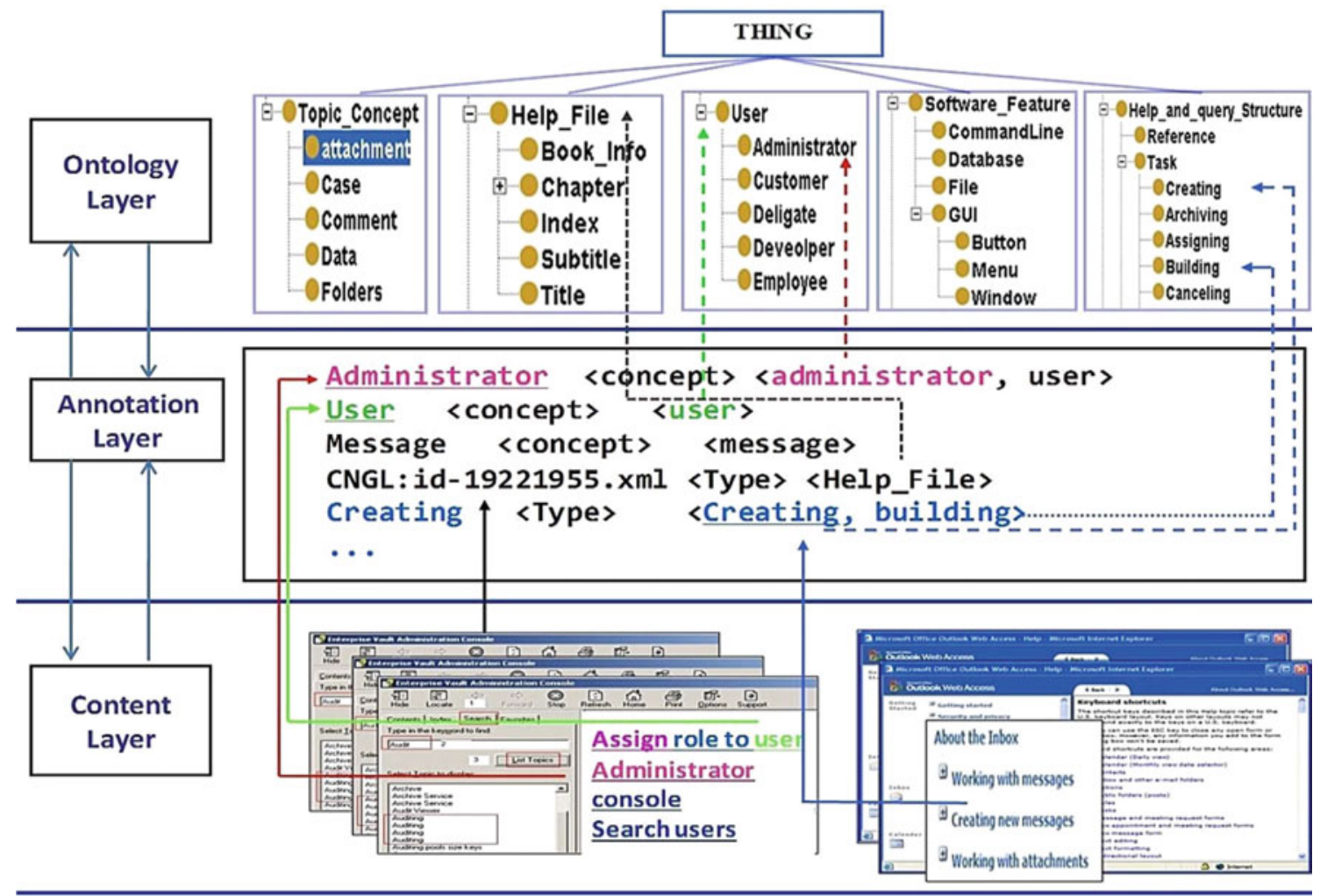

Fig. 2 Ontology-driven content-based systems (ODCBS)

FSG [30] algorithm. The performance study shows that the gSpan outperforms the FSG algorithm and is capable of mining large frequent subgraphs. The fast frequent subgraph mining (FFSM) algorithm [20] is an algorithm for graph-based pattern discovery. FFSM can be applied to protein structures to derive structural patterns. Their approach facilitates families of proteins demonstrating similar function to be analyzed for structural similarity. Compared with gSpan [49] and FSG [30] algorithms using various support thresholds, FFSM is an order of magnitude faster. gSpan is more suitable for small graphs (with no more than 200 edges).

We adopted ideas from sequential pattern discovery approaches in other domains, such as sequence-independent structure pattern [20] and gap-constraint [31] for setting cutoffs in terms of node matching mechanism. Yet, discovery of change patterns from ontology change logs is relatively different from sequential pattern discovery in other contexts (such as the biomedical domain). Recently, few researchers have focused on detection of higher level generic ontology changes $[14,35]$. In contrast to their work, our approach is to discover the change patterns and is based on contextaware, semantic matching of different graph sequences. This requires the identification of equivalency between unordered change sequences.

Our work regarding the formalization and storage of the discovered change patterns is relatively similar to the work of Henninger [19] and Kampffmeyer [26]. Similar to our generic metadata change ontology, Henninger uses ontology-based metamodels to formally present the software patterns. The core properties of the metamodels include hasProblem, hasSolution, hascontext, etc. To represent the relationship among the patterns, the core metamodel was extended using properties uses, requires, alternatives, etc. In contrast to our work, the focus of their ontology-based metamodel is to represent the relationships between the software patterns, rather than providing support of selecting the suitable pattern for a given task. In [26], author proposed a Design Pattern Intent Ontology (DIPO) for formalizing the patterns, based on their intent. The aim of DIPO is to support the software developers in choosing a design pattern, suitable for a particular task.

\section{Ontology Change Management}

We have been working with non-public ontologies used to annotate the content in large-scale information systems. The aim here is to facilitate accessibility of content for both humans and machines by integrating semantics in the content using ontologies. In this regard, a content change will ultimately affect all the artefacts - the access files being updated, the information system entities being improved. The latter causes knock-on effects on the access files and also the ontology-driven content management model (Fig. 2). 
Fig. 3 Layered framework of change operators and patterns

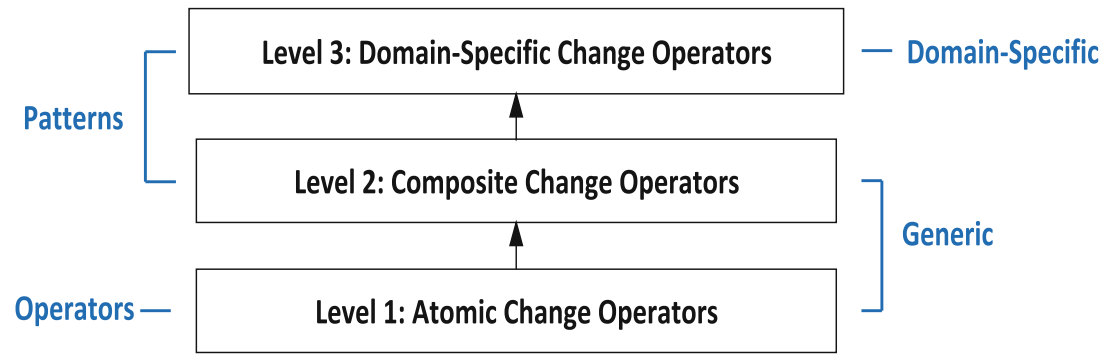

We distinguish two categories of changes - changes to the content artefacts (content management infrastructure artefacts) and changes to the ontologies as the knowledge on top of the artifact layer [15].

- Ontological Changes can include changes in the concept hierarchy; some concepts may get modified, removed, pulled up/down in the hierarchy, etc. More description (in a form of object/data properties) can be added to the available concepts. Ontological changes could reflect the general changes in the domain, flaws in the earlier conceptualization, addition of new concepts in the domain, etc.

- Content Changes can affect any of the artifacts. Particular interest here is the cascading impact. A content change may have direct impact on access files. A change in access file may have direct impact on the annotations that link the files to the domain ontologies; and thus the underlying domain ontology may also evolve accordingly.

In this paper, we focus on ontological changes only. We propose a four-phase ontology change management system (Fig. 1). These phases include, change operationalisation (phase-1), change representation (phase-2), change semantic capturing (phase-3) and change pattern discovery (phase4 ). In the following sections, we discuss each phase one after the other.

\section{Layered Change Operator (LCO) Framework}

Based on an empirical observation of common changes in different ontologies, we defined a layered framework of change operators (Fig. 3). The first two layers are based on generic and structural change operators. The next layer covers domain-specific changes. We consider level two and three change operators as "change patterns".

We define a change pattern as a frequently occurring composed operation. The change pattern represents a frequently occurring constrained composition of lower-level change operations over the ontology elements. The main difference

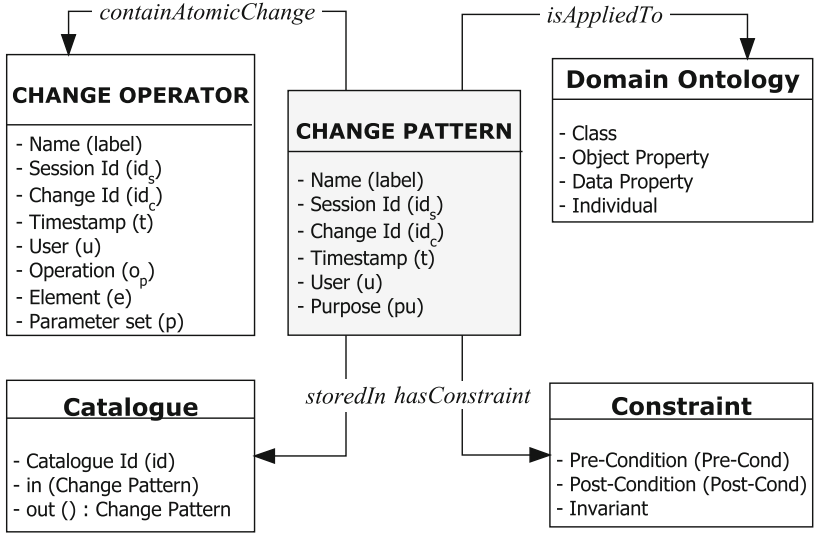

Fig. 4 Structural model of pattern-based ontology evolution

between a pattern and a composite operation is the frequency. It is the result of a mining process, whereas composite operations are language elements. These change patterns can either be generic or domain-specific. A generic change pattern can be applied to any domain ontology, whereas a domain-specific change pattern can only be applied to a specific domain ontology. The structural model of pattern-based ontology evolution is given in Fig. 4.

Level one change operators-atomic change operations: These change operators are the elementary change operations used to perform a single add or delete operation on a single targeted entity (i.e., addition or deletion of any particular axiom in the ontology). "Add classDeclarationAxiom(Student)", "Delete subClassOfAxiom(Student, Person)", etc., are the examples of level one change operations.

Level two change operators composite change patterns: These are aggregated changes to represent composite tasks. Many evolution tasks cannot be done by a single atomic operation. These change operators are identified by grouping atomic operations of level one to perform a composite task on target entities.

Composite change operations comes into two layers. First layer of composite change operations includes group of those atomic change operations that in general are executed 


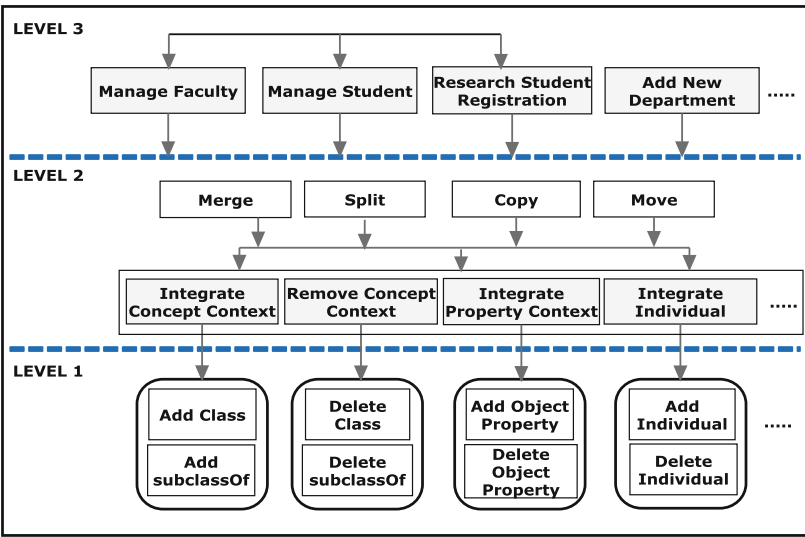

Fig. 5 Architecture of layered change operators (University Ontology)

together. For example, "Remove Concept Context" which not only deletes a concept from the class hierarchy, but also deletes all its roles. To delete a single concept "faculty" in a university ontology, removing the concept from the concept hierarchy is not sufficient. Before we remove the concept, we have to remove all its roles, such as removing from the domain and the range of properties like "isSupervisorOf" or "hasPublication", etc. In addition, we need to either delete its orphaned subclasses or link them to the parent concept, in order to keep the ontology consistent.

If an ontology engineer wants to merge two or more concepts, the operation requires operators higher than the integrate/remove concept context. In such a case, composite change operations from level two can be used. "merge concepts", "move concepts" or "pull up property" are examples of layer two composite change operations.

Level three change operators domain-specific change patterns: The changes at a higher level of granularity, which are frequent in a domain, can be represented as domainspecific patterns-which are often neglected by the lowerlevel compositional change operators. Domain-specific perspective links the structural changes to the aspects represented in domain ontologies. To execute a single domainspecific change, operations at level one and two are used. In case of the university administration domain, level three may contain change patterns such as "manage faculty", "add new department", "student registration", etc. (Fig. 5). If a user needs to register a new category of faculty using the "manage faculty" change pattern, say a "JuniorLecturer", then he creates a concept "JuniorLecturer" and attaches properties like "hasPublication" and "supervise" from level two to the newly created concept. Another ontology engineer may create a new concept "JuniorLecturer" without including the "supervise" property. This is due to the different viewpoints and perspectives of the users.

More details about the change operator framework can be found in [22].

\section{Layered Change Log Representation}

Ontology change logs can play a significant role in ontology evolution. If there is a need to reverse a change, we use the change log to undo/redo the changes applied in the past. This is a common function in e.g. software versioning support. In collaborative environments, change logs are also used to keep the evolution process transparent and centrally manageable. It captures all changes ever applied to any entity of ontology using elementary changes. We propose a mechanism of representing ontology changes expressively at different levels of granularity (i.e. fine-grained changes such as the creation of a single class and also coarse-grained changes such as merging two sibling classes [38]). The higher-level change representation is used for

- describing how an ontology is evolved from one version to the other.

- In distributed environment where complex relationships can exist between domain ontologies and other artefacts, an ontology change may need to propagate to dependent artefacts. In such cases, higher level change representation assists in understanding the ontology change and the impact (consequence) of the applied changes.

- bridging between operational and analytical aspects of the ontology evolution.

\subsection{Layered Change Log Model}

Capturing and representing the ontology changes at the elementary level in a change log do not suffice. As the intent of the ontology change is missing from such change logs (and mostly specified at higher level of granularity), the ontology engineer is unable to understand why changes were performed, whether it is an elementary level change or a part of composite change and what the impact of such change is. We attempt to mine valuable information from a change $\log$, making it easy for the ontology engineer (other) users and machines to understand and interpret the ontology modifications. We propose a layered change log model, containing two different levels of granularity, i.e. an atomic change log (ACL) and a pattern change log (PCL), shown in Fig. 6. The

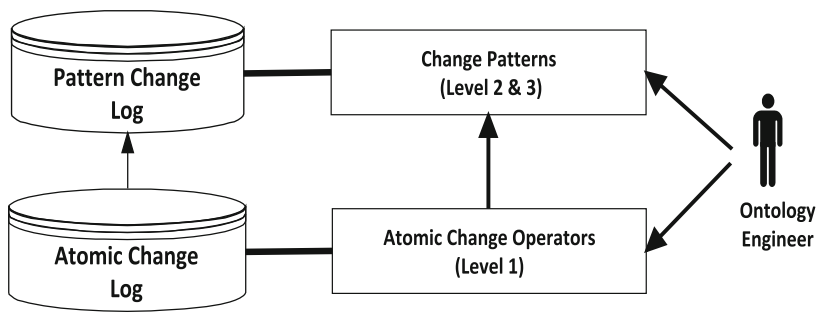

Fig. 6 Layered ontology change framework 
Fig. 7 Representation of an atomic ontology change
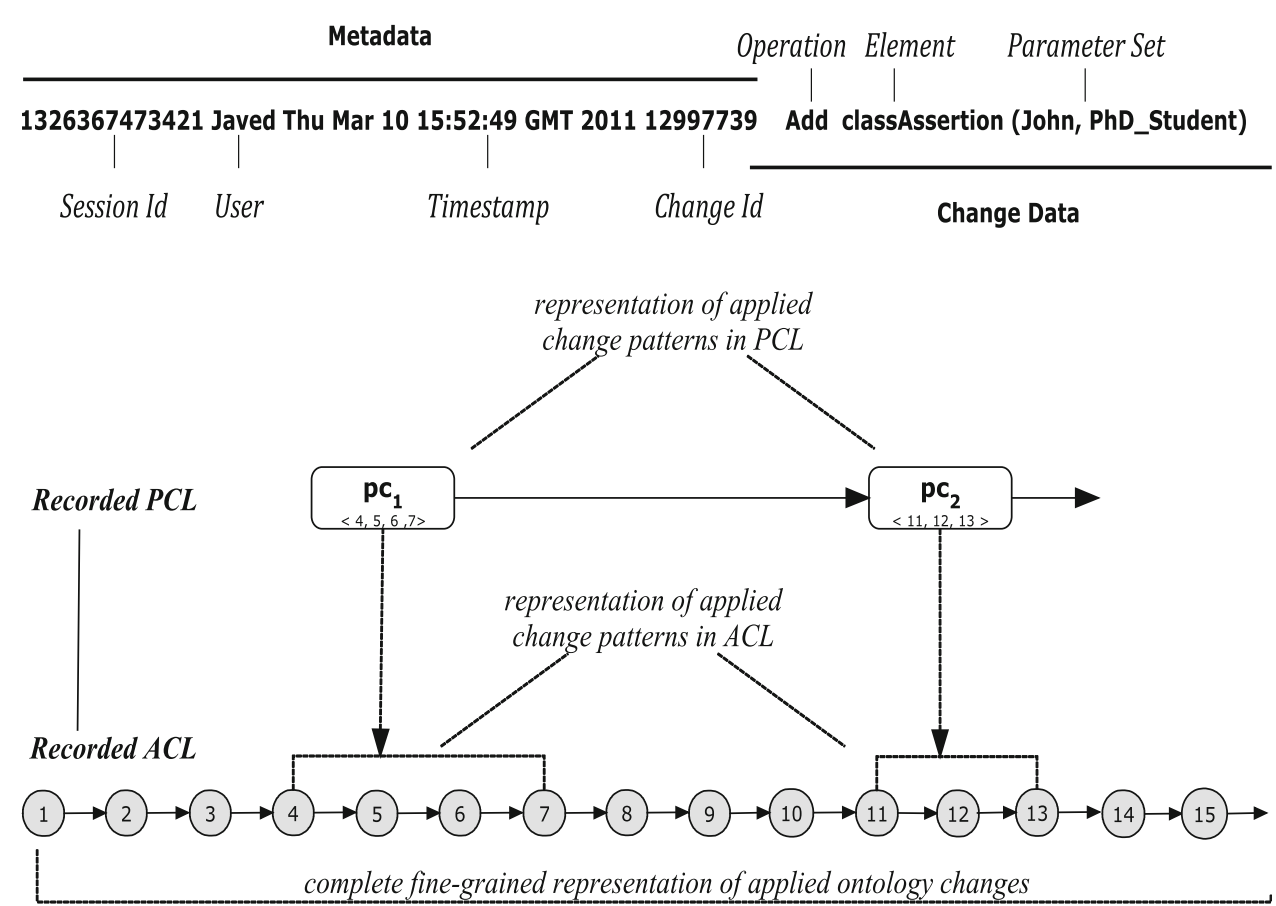

Fig. 8 Operational setup of ontology change logging
Pattern change $\log$ A PCL consists of an ordered list of ontology change patterns, $\mathrm{PCL}=<p c_{1}, p c_{2}, p c_{3} \cdots p c_{n}>$ where $n$ refers to the sequence of ontology change patterns in a PCL. These change patterns can either be level two generic composite change patterns or level three domainspecific change patterns (cf. Fig. 5). Similar to ACL, each ontology change pattern $p c$ consists of two types of data i.e. Metadata $(D)$ and Pattern data $(P)$. The metadata provides meta details about the change pattern and can be given as $D=\left(i d_{s}, i d_{c}, u, t, p_{u}\right)$ where, $i d_{s}, i d_{c}, u, t$ and $p_{u}$ represent the session id, change id, user, timestamp and intention of the change pattern, respectively.

The pattern data $(P)$ provide description about the involved change operations. Here, $P$ refers to the sequence of the change operations available in a change pattern $P=$ $\left(a c_{1}, a c_{2}, \ldots a c_{s}\right)$, where $s$ is the total number of change operations in a pattern. For a complete representation of applied ontology changes, the applied change patterns are recorded as a sequence of atomic change operations in the ACL (Fig. 8).

\subsection{RDF Framework Format}

We use RDF triple-based representation, i.e., subjectpredicate-object (spo), to conceptualize the ontology changes in change logs. To do so, we constructed a generic metadata ontology ${ }^{1}$ based on specification of OWL-DL 2.0. The classes and properties available in the metadata ontology assist the ontology engineer to construct the RDF triples,

\footnotetext{
${ }^{1}$ Available at www.computing.dcu.ie/ mjaved/MO.owl
} 
Namespaces: MO: $\quad$ http://www.cnglie/ontology/MO.owl\# University: http://www.cngl.ie/ontology/University.ow\#\#

1. 〈MO:ResearchStudentRegistration><rdf:type > $<\mathrm{mo}$ :PatternChange $>$

2. <MO:ResearchStudentRegistration> <MO:Sessionld > "7536902801513"

3. <MO:ResearchStudentRegistration> $<M O$ :changeld $>$ "1323865264484"

4. $\langle\mathrm{MO}$ :ResearchStudentRegistration $>\langle\mathrm{MO}$ :hasCreator $>\langle\mathrm{MO}:$ :Javed $>$.

5. <MO:ResearchStudentRegistration><M0:Timestamp> "Wed Dec 14 12:21:04 GMT 2011".

6. 〈MO:ResearchStudentRegistration> $<M 0:$ PatternName $>$ "PhD Student Registration" .

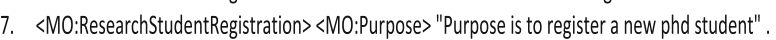

8. $\langle M O$ :ResearchStudentRegistration $>\langle M O$ :containAtomicChange $>\langle M O$ :AddIndividual $>$.

9. $\langle M O$ :ResearchStudentRegistration $>\langle M O$ :containAtomicChange $>\langle M O: A d d I n d i v i d u a l T y p e>$.

10. $<M O$ :ResearchStudentRegistration $><M O$ :containAtomicChange $><M O$ :AddObjectPropertyAssertion

11. $\langle\mathrm{MO}$ :Addlndividual $><$ dff:type $><\mathrm{MO}$ :AtomicChange $>$.

12. $\langle\mathrm{MO}:$ AddIndividual $><\mathrm{MO}$ :changeld $>$ "13238652644840"

13. $\langle M O$ :AddIndividual $><M O$ :hasEntity $><M O:$ Individual $>$

14. $<M O$ :Addlndividual $><M O$ :hasTargetParam $><M O$ :param1 $>$.

15. $\langle M O$ :Addlndividual $>\langle M O$ :hasOperation $>\langle M O: A d d\rangle$.

16. 〈MO:AddlndividualType >rdf:type > $<\mathrm{MO}$ :AtomicChange>.

17. 〈MO:AddIndividualType $><M O: c h a n g e l d>" 13238652644841 "$.

18. $<M 0:$ AddIndividualType $>M M O$ :hasAuxParam1 $1<\langle$ University:PhD_Student>

19. 〈MO:AddIndividualType $>\langle M O$ :hasIndividualAxiom $>\langle M O$ :classAssertionAxiom $>$.

20. $\langle$ MO:AddlndividualType $>$ $<\mathrm{MO}$ :hasTargetParam $>\langle$ University:param1 $>$.

21. $\langle\mathrm{MO}$ :AddIndividualType $>\langle\mathrm{MO}$ :hasOperation $>\langle\mathrm{MO}: \mathrm{Add}\rangle$.

Fig. 9 RDF triple-based specification of stored change pattern "ResearchStudentRegistration"

representing an applied ontology change. Similar to the approaches opted for [34] and [36], the idea here is to provide a metadata ontology that is generic, independent and extendable to represent the changes of the domain ontologies. We used an RDF triple store to record the change log, domain ontologies and metadata ontology. Thus, all ontology changes, stored in the ontology change log, are in a form of triples. Below, we give description of the metadata ontology, using an example of stored change pattern, given in Fig. 9.

The central class in the metadata ontology is Change. Based on our proposed change operator framework (cf. Sect. 4), the class Change is subdivided into AtomicChange, CompositeChange and PatternChange. Each stored domainspecific change pattern is an instance of (rdf:type) PatternChange (line 1: Fig. 9). The descriptive data of a change pattern are given using properties sessionId, changeId, PatternName, Timestamp, Purpose, etc. (lines 2-7). To express that the change pattern is the combination of lower level change operations (i.e. atomic, composite or combination of them), the class PatternChange is associated with the class AtomicChange and the class CompositeChange using object properties containAtomicChange and containCompositeChange, respectively (lines 8-10).

\subsection{Graph-based Ontology Change Formalization}

Ontology change logs provide operational as well as analytical support in the evolution process. As discussed in Sect. 5.2, the ontology change logs are stored in the form of RDF triples. RDF triple format is used due to its fine-grained level representation and interoperability (i.e., conversion from triple format to others standard formats such as RDF, $\mathrm{XML}$, etc.). Fine-grained representation of ontology changes help the ontology engineer to construct complex queries and extract different types of knowledge from the log. Furthermore, storing domain ontologies, metadata ontology and change $\log$ in one single location help in navigating through them simultaneously and identifying relationships among them. However, as RDF triples represent the ontology changes at fine-grained level ( 1 ontology change is represented by $8-10$ triples), efficiently visualizing and navigating through the change log alone is not realistic. Graphs can cover this gap. Graphs provide the ability to visualize and navigate through large network structures. They enable efficient search and analysis and can also communicate information visually. Moreover, the benefit of a graph-based representation is the availability of well-established algorithms/metrics (for pattern discovery and detection) and its well-known characteristics such as performance (for querying the ontology change effectively).

A graph-based formalization is an operational representation for the ontology changes. To identify the higher level change patterns from the ACL, we reformulate the triplebased representation of atomic changes using a graph-based approach. We use attributed graphs [11]. Graphs with node and edge attribution are typed over an attribute type graph (ATG). Attributed graphs (AG) ensure that all edges and nodes of a graph are typed over the ATG and each node is either a source or target, connected by an edge (Fig. 10). The benefit of using ATGs and AGs is their similarity with the object oriented programming languages, where one can assign each element of the graph a type. Similar to the objects of any class, having a number of class variables, one can attach a number of attributes to a graph node in an AG. The data types of such attributes can be defined in an ATG. Furthermore, one can borrow other object oriented concepts, such as inheritance relations, for any defined element in an ATG.

Based on the idea of attributed graphs, a change log graph $G$ can be given as $G=\left(N_{G}, N_{A}, E_{G}, E_{N A}, E_{E A}\right)$ where:

- $N_{G}=\left\{n_{g}^{i} \mid i=1, \ldots, p\right\}$ is the set of graph nodes. Each node represents a single ontology change log entry (i.e., representing a single atomic ontology change). The term $p$ refers to the total number of atomic change operations present in the ACL. Here, we assume that the concurrent 
Fig. 10 Attribute type graph (ATG) for an ontology change

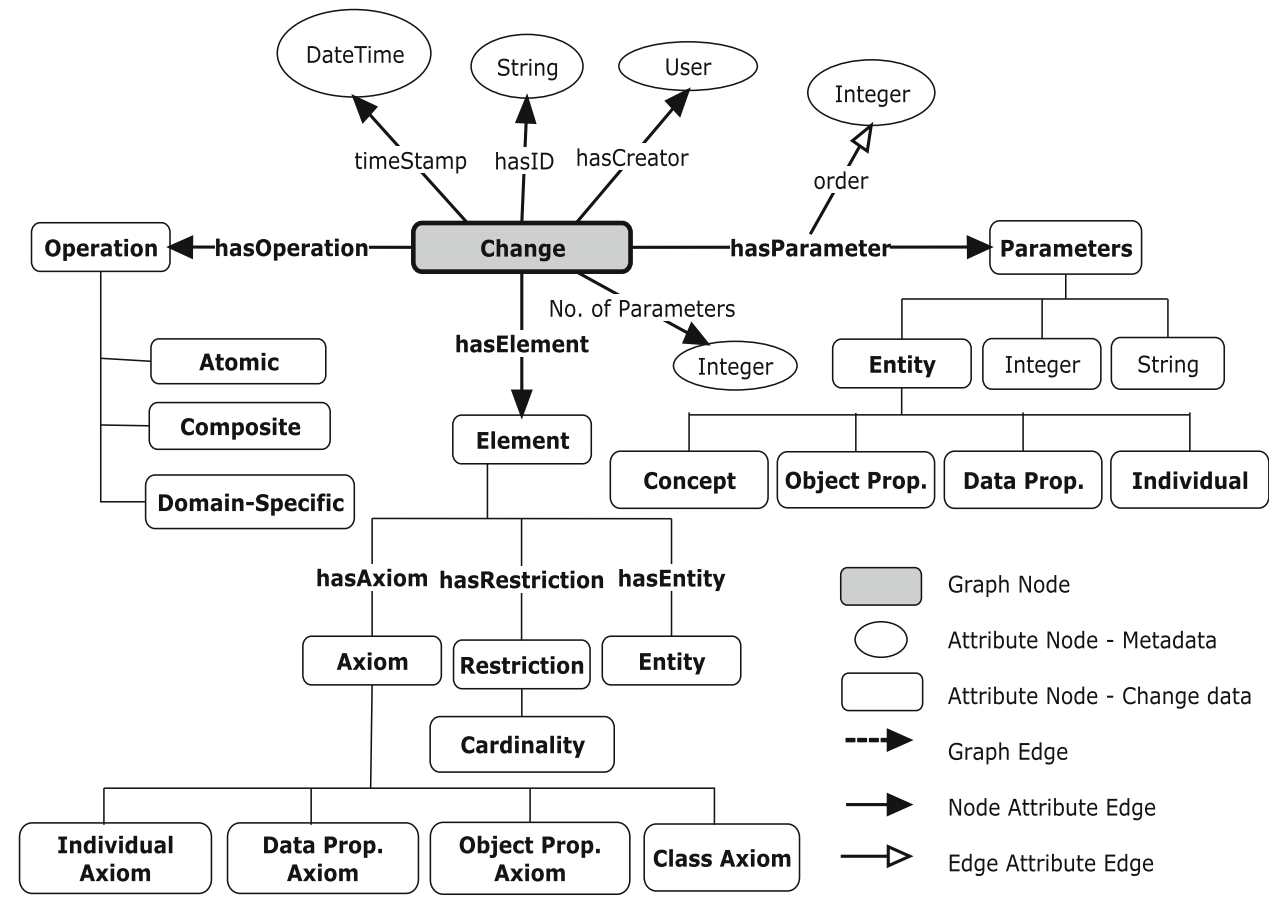

ontology change operations (if any) are sequenced; i.e., each ontology change operations is executed one after another.

- $N_{A}=\left\{n_{a}^{i} \mid i=1, \ldots, q\right\}$ is the set of attribute nodes. Attribute nodes are of two types: (1) attribute nodes which symbolize the metadata (e.g. change Id, user, timestamp) and (2) attribute nodes which symbolize the change data (and its subtypes) (e.g. operation, element, target parameter, auxiliary parameters) —cf. Fig. 7. The term $q$ refers to the total number of attributes attached to a single graph node $n_{g}$.

- $E_{G}=\left\{e_{g}^{i} \mid i=1, \ldots, p-1\right\}$ is the set of graph edges which connects two graph nodes $n_{g}$. The graph edges $e_{g}$ represent the sequence of the ontology change operations in which they have been applied on the domain ontology.

- $E_{N A}=\left\{e_{n a}^{i} \mid i=1, \ldots, r\right\}$ is the set of node attribute edges which joins an attribute node $n_{a}$ to a graph node $n_{g}$.

- $\left.E_{E A}=\left\{e_{e a}^{i}\right) \mid i=1, \ldots, q-r\right\}$ is the set of edge attribute edges which joins an attribute node $n_{a}$ to a node attribute edge $e_{n a}$.

A single graph node of an attributed graph (AG) which is typed over an ATG is given in Fig. 11. The types defined on the (graph and attributed) nodes can be given as $t(A d d)=$ Operation, $t$ (class Assertion $)=$ Element , $t(J o h n)=$ Individual and $t\left(P h D \_S t u d e n t\right)=$ Concept . This node represents a single ontology change operation where graph node $n_{g}$ is the central part of it. These graph nodes are linked to each other using graph edges $e_{g}$ to represent a complete ontology change log graph.

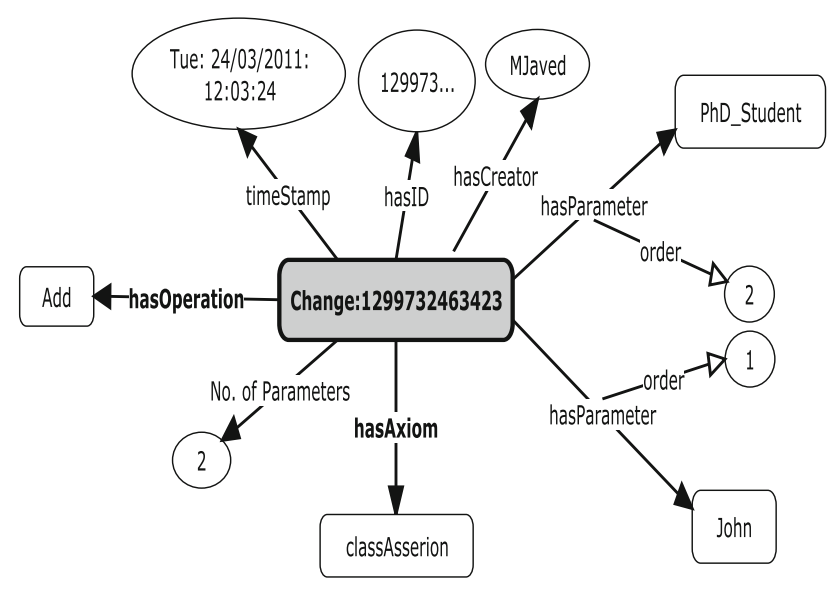

Fig. 11 Attributed graph Node typed over ATG (Add classAssertion (John, PhD_Student))

\section{Detection of Composite Change Patterns}

As we discussed in Sect. 5, representing a change at the atomic level is not sufficient. Such representation of ontology changes can only describe the addition or deletion of an ontology element. The semantics of an applied change (or a group of changes) are missing from such representation and most of the time is present at higher level of granularity. We can represent such semantics of the applied atomic changes in the form of composite change patterns and can be represented in the PCL. For example, if a concept $x$ is removed (as a subclass) from a parent concept $y$ and has been attached (as a subclass) to another concept $s$, the semantics behind 


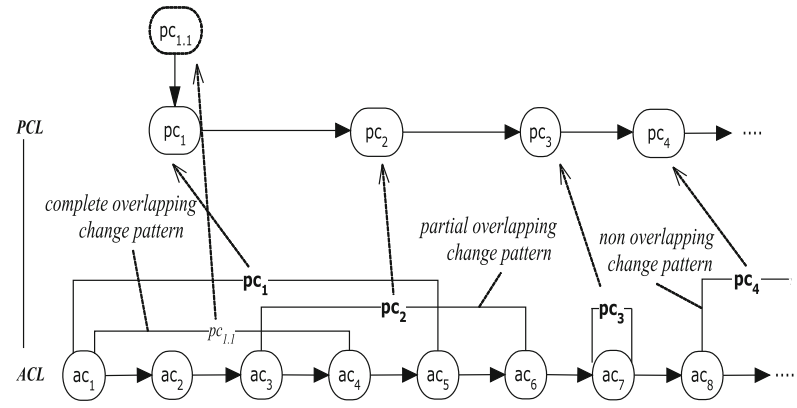

Fig. 12 Layered change log framework

such change (at atomic level) only refers to a change of the class hierarchy for concept $x$, i.e. Move Concept (x, $\mathrm{y}$, s). In this case, instances of concept $x$ that inherit properties from concept $y$ or any of its parents need to be revalidated. However, if we identify this knowledge that concept $s$ is actually a superclass of concept $y$, the semantics behind such a change will refer to a "pull up concept" change-Pull up Concept (x, y, s). In this case, instances of concept $x$ that inherit properties of concept $y$ only need to be revalidated. This example signifies the importance of capturing the semantics of any change at a higher level. We operationalize the change semantic captured using composite change detection algorithms. The algorithms lead us to the detection of composite changes from ACLs and their (semantically) enhanced representation in the PCL. It is common to find some overlapping change patterns. Such overlapping of the change patterns can be either complete or partial (Fig. 12).

\subsection{Composite Change}

A composite change is a sequence containing a group of elementary (level one) change operations that are applied on a domain ontology, where the change operations can be of inclusion or exclusion type. The inclusion type change operations add new data to the domain ontology, whereas the exclusion type change operations remove data from the domain ontology. Thus, a composite change $c$ can be given as $\left\langle\delta_{1}, \delta_{2}, \phi>\right.$, where:

- $\delta_{1}$ is a sequence of atomic level exclusion change operations.

- $\delta_{2}$ is a sequence of atomic level inclusion change operations.

- $\phi$ refers to the conditions to be satisfied.

As composite change operations are applied at the entity level, an exclusion change operation $\left(\delta_{1}\right)$ deletes certain axioms from the target ontology entity. The inclusion change operation $\left(\delta_{2}\right)$ adds some new axioms regarding the target ontology entity. Further, to consider a group of (add/delete) change operations as a composite change, the change operations must satisfy certain conditions. The term $\phi$ refers to the conditions on the existence of any knowledge in the ontology. Such conditions can be either existential conditions $\left(\phi_{e}\right)$ or correlations $\left(\phi_{c}\right)$ among the ontology change parameters. The existential conditions $\left(\phi_{e}\right)$ of any change operation can be given in terms of pre and post conditions. For example, in case of change operation Add concept (Researcher), the individual Researcher must not exist in the current version of ontology $\left(O_{1}\right)$ and must exist (as a concept) in the next version of ontology $\left(\mathrm{O}_{2}\right)$.

- Pre-Cond: Researcher $\notin O_{1}$

- Post-Cond: Researcher $\in O_{2}$

- Post-Cond: (Researcher rdf:type owl:Class) $\in \mathrm{O}_{2}$

Similarly, in case of Add subclassOf (Researcher, Person), concepts Researcher and Person must exist in the current version of ontology $\left(O_{1}\right)$ and Researcher should be a child of (rdfs:subClassOf) Person in subsequent version of ontology $\left(\mathrm{O}_{2}\right)$.

- Pre-Cond: Researcher, Person $\in O_{1}$

- Post-Cond: (Researcher rdfs:subClassOf Person) $\in \mathrm{O}_{2}$

The correlations $\left(\phi_{c}\right)$ refer to the relationships among the parameters of the available atomic change operations in a composite change. Such relationships are not explicitly given in the ACL. For example, in case of composite change operation Pull up concept (Researcher, Student), where the concept Researcher is being pulled up in the concept hierarchy and becomes a sibling class to its previous parent concept Student, the change is actually a group of two atomic change operations, i.e.,

- Delete subclass of (Researcher, Student). $\left(\delta_{1}\right)$

- Add subclassof(Researcher, Person). $\left(\delta_{2}\right)$

the correlations can be given as

- Student subclassof Person. $\left(\phi_{c}\right)$

We utilized the given definition of a composite change in defining the graph transformation rules and conditions. In other words, we can say that a source ontology subgraph has been transformed into target ontology subgraph based on the given conditions, i.e., existential and correlation conditions.

\subsection{Graph-based Specification of a Ontology Change}

We specify the ontology changes of composite types using a graph transformation approach where a source ontology subgraph is transformed into a target ontology subgraph, while preserving the defined conditions. We opt for the double pushout (DPO) [10] approach that allows us to specify the graph transformation rules and gluing conditions in a 


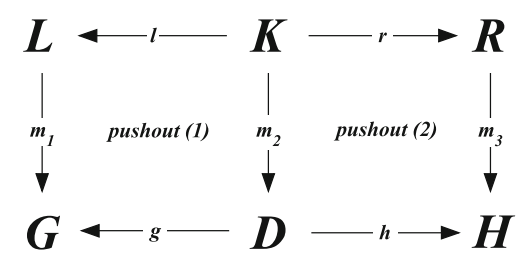

Fig. 13 Double-pushout approach for graph transformation

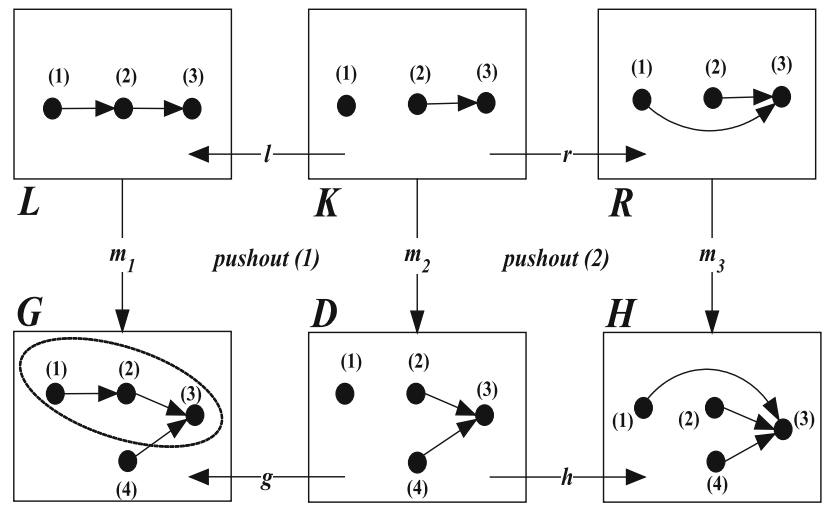

Fig. 14 DPO approach — an example

form of pairs of graph morphisms $(L \stackrel{l}{\leftarrow} K \stackrel{r}{\rightarrow} R)$-Fig. 13 . First, we describe the DPO approach.

Referenced vs. Ontology subgraphs The DPO approach is termed as "double pushout" as the complete transformation of input ontology subgraph $G$ into target ontology subgraph $H$ is translated into two types of changes, i.e., exclusion and inclusion change operations. The DPO approach uses a graph mapping approach where subgraphs $L, K$ and $R$ represent the referenced subgraphs and subgraphs $G, D$ and $H$ represent the ontology subgraphs. Thus, we can say that the ontology subgraphs $G, D$ and $H$ are mapped to referenced subgraphs $L, K$ and $R$, respectively (Fig. 14).

The graph $L$ is the referenced input subgraph representing items (i.e., nodes or edges) that must be present in the ontology input subgraph $G$ for the application of the composite change. In other words, graph $G$ represents the initial state of the ontology. The graph $R$ is the referenced output subgraph representing the items that must be present in the resulting target ontology subgraph $H$, after the application of the composite change, i.e., representing the final state of the ontology; whereas, the referenced graph $K$ represents the "gluing graph" $(L \cap R)$, also known as interface graph, representing the graph items that must be read during the transformation but are not consumed, i.e., representing the intermediate state after the application of exclusion type atomic change operations. Note, the graph transformation here represents the transformation of an input ontology subgraph into a target ontology subgraph — not the transformation of a "change log" subgraph. Each node in a DPO graph represents an ontology entity (i.e. class, property or individual). In Fig. 14, the nodes and the edges represent the ontology classes and the subclassOf axioms, respectively. The change log graphs are mentioned here in the form of productions and co-productions (discussed below), representing the set of atomic change operations.

The graph transformation rules, also known as productions $(p)$, represent the change operations being applied on the subgraphs during the two pushouts. The rules define the correspondence between the source and the target subgraph determining what is to be deleted, preserved or constructed. For example, in Fig. 13, first production, represented as $l$, refers to the exclusion change operations of pushout 1 that delete certain items (nodes or edges) from the reference input subgraph $L$. The second production, represented as $r$, refers to the inclusion change operations of pushout 2 that adds certain items (nodes or edges) into the reference gluing graph $K$. The productions representing the changes being applied on the input ontology subgraph $G$ are known as co-productions and are given as $g$ and $h$ in Fig. 14.

Match $(m)$. To apply production $l$ to the ontology graph, first we need to identify the occurrence of subgraph $L$ in the ontology graph, called a "match". For example, $m_{1}: L \longrightarrow$ $G$ for a production $l$ is a graph homomorphism, i.e., each node/edge of graph $L$ is mapped to a node/edge in graph $G$ in such a way that graphical structure and labels are preserved [6]. The context gluing graph $D$ is obtained by deleting all items (nodes and edges) from the subgraph $G$ which have a match (image) in the subgraph $L$ but not in subgraph $K$ (pushout-1). Intuitively, we can say that if a match $m_{1}$ finds an occurrence of subgraph $L$ in a given ontology subgraph $G$, then $G \stackrel{l, m_{1}}{\Longrightarrow} D$ represent the derivation (co-production) $g$ where $l$ is applied to $G$ leading to a derived graph $D$. Informally, the subgraph $D$ is achieved by replacing the occurrence of $L$ in $G$ by $K$. Similarly, in pushout-2, the subgraph $H$ is obtained by inserting distinct items (nodes and edges) of subgraph $R$ thats do not have any match (image) in subgraph $K\left(h=D \stackrel{r, m_{2}}{\Longrightarrow} H\right)$.

Gluing conditions The possible conflicts in the graph matching step are resolved by applying certain matching constraints, known as "gluing conditions". A gluing condition consists of two parts, i.e., a dangling condition and an identification condition. The dangling condition $\left(C_{d}\right)$ ensures that the graph $D$, obtained by applying the production $l$, contains no "dangling" edge, i.e., an edge without a source or a target node. For example, if a node $v$ is deleted from graph $G$, all the edges that contain node $v$ as a source or target node, will also be deleted. The identification condition $\left(C_{i}\right)$ ensures that every item of graph $G$ that has to be deleted by the application of production $l$, must have only one distinct match in the graph $L$, i.e., 1:1 matching. Thus, we can say that the items from the left hand side graph $L$ may only be identified in 


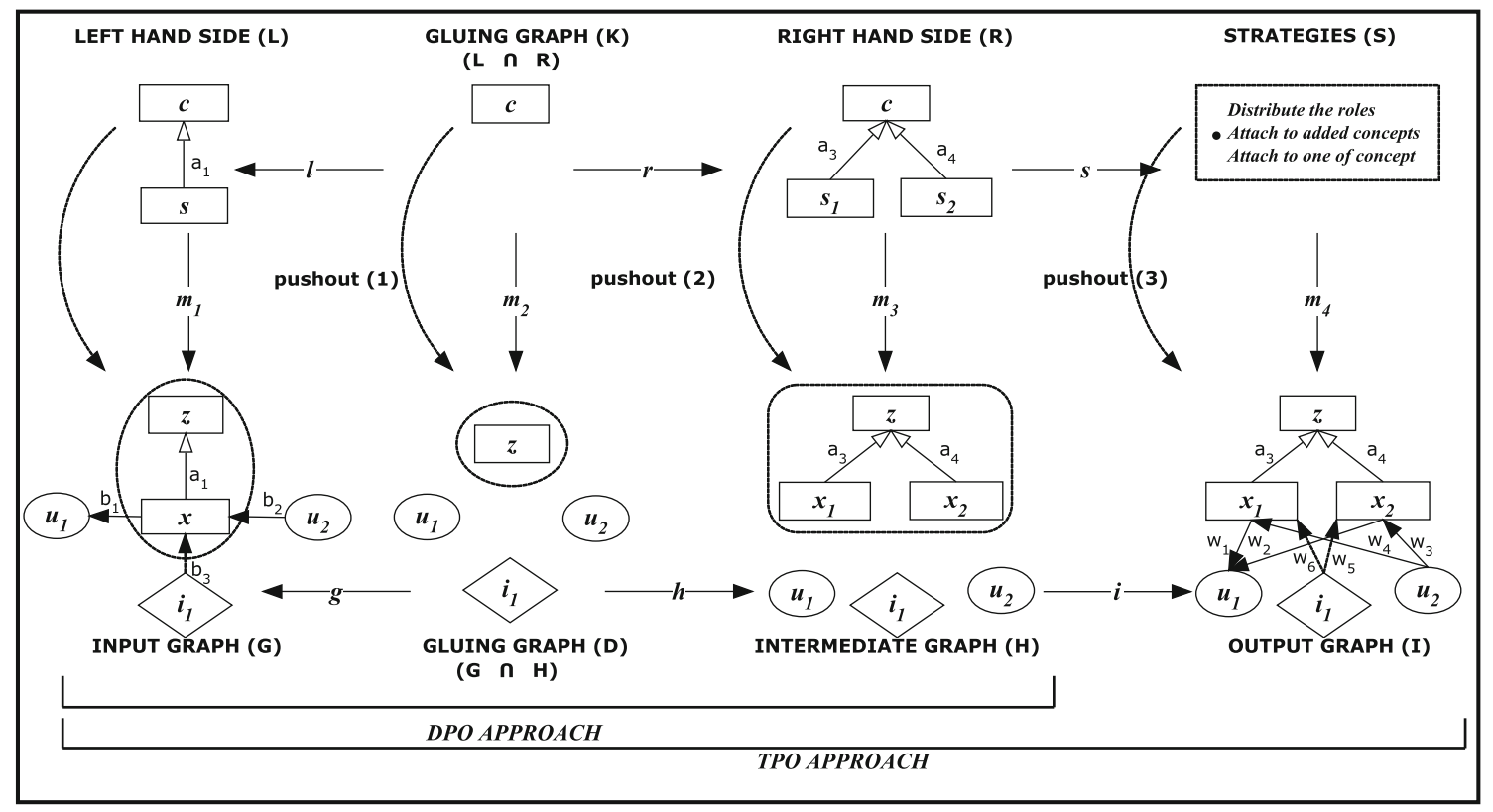

Fig. 15 Split concept $\left(x,\left(x_{1}, x_{2}\right)\right)$-triple push out (TPO) approach

resultant graph $R$ if they also belong to the gluing graph (i.e., preserved items) [18].

\subsection{Triple Pushout (TPO) Approach}

We take pushouts 1 and 2 of DPO approach as "structural pushouts", as they refer to completeness and correctness of the structure of a graph. The dangling condition for edges in pushout 1 of DPO approach does ensure that the graph $D$ is a genuine graph by deleting the dangling edges. However, the semantics behind the applied composite change may be lost, e.g. in the case of split concept change. Let $x$ be an ontology concept that is split into two concepts $x_{1}$ and $x_{2}$ (Fig. 15). In pushout 1 of split concept change, concept $x$ is deleted from the concept hierarchy. To satisfy the dangling condition, the roles (edges) of the concept $x$ are also being deleted. In pushout 2, two new concepts $x_{1}$ and $x_{2}$ are added replacing concept $x$ in the concept hierarchy. As concepts $x_{1}$ and $x_{2}$ inherit relationships from the split concept $x$, the deleted roles (edges) are not the consumed entities in this graph transformation. Thus, the relationships must be added back to the newly added concepts $x_{1}$ and $x_{2}$.

To do so, we extended the DPO approach by adding an additional production that formulates the pushout 3 (semantic pushout) allowing a user to preserve the deleted dangling edges of pushout 1 ; hence, named "triple pushout approach". In pushout 3, the user can select different evolution strategies [25] to resolve the above mentioned issues. Thus, a derivation $i$ from subgraph $H$ to $I$ resulting from an application of production $s$ (pushout 3) at a match $m_{3}$ can be given as $i=$ $H \stackrel{s, m_{3}}{\Longrightarrow} I$ (Fig. 15). Now, we explain the TPO approach in detail using the "split concept" composite change case scenario.

"Split concept" Change Scenario The composite change "split concept" refers to splitting a concept into two (or more) sibling concepts. For example in Fig. 15, the concept $x(x \in G)$ has been split into two sibling concepts $x_{1}$ and $x_{2}\left(x_{1}, x_{2} \in I\right)$. The composite change adds two new concepts in the ontology (inclusion operations) and deletes the concept that has been split (exclusion operation). The nodes and edges, given in Fig. 15, represent the following ontology elements: square node $\longrightarrow$ concept (c), oval node $\longrightarrow$ property $(\mathrm{t})$, diamond node $\longrightarrow$ individual (i), edge $[\operatorname{src}(\mathrm{e})=\mathrm{c} \&$ $\operatorname{tar}(\mathrm{e})=\mathrm{c}] \longrightarrow$ is-a relationship, edge $[\operatorname{src}(\mathrm{e})=\mathrm{t} \& \operatorname{tar}(\mathrm{e})=$ c] $\longrightarrow$ range of a property, edge $[\operatorname{src}(\mathrm{e})=\mathrm{c} \& \operatorname{tar}(\mathrm{e})=\mathrm{t}] \longrightarrow$ domain of a property and edge $[\operatorname{src}(\mathrm{e})=\mathrm{i} \& \operatorname{tar}(\mathrm{e})=\mathrm{c}] \longrightarrow$ instanceOf relationship.

Table 1 gives the formal definition of the split concept composite change example given in Fig. 15, in terms of ontology and TPO graph changes and conditions. Now we discuss each pushout and the involved change operations one after the other.

pushout 1 First, we identify the occurrence of the reference subgraph $L$ in ontology graph (i.e., $m_{1}: L \longrightarrow G$ ). Once the match is found, production $l$ is being applied to the matched ontology subgraph $G$ (through co-production $g$ ) resulting in a gluing graph $D$ (i.e., $g=G \stackrel{l, m_{1}}{\Longrightarrow} D$ ). The co-production $g$ represents the deletion of concept $x$ from the ontology concept hierarchy. Thus, in Fig. 15, node $x$ and edge $a_{1}$ are deleted from the input ontology subgraph $G$. Furthermore, to satisfy the dangling conditions, edges $b_{1}, b_{2}$ and $b_{3}$ are also deleted. 
Table 1 Formal definition of composite change operation: Split Concept $\left(x,\left(x_{1}, x_{2}\right)\right)$

Split concept $\left(x,\left(x_{1}, x_{2}\right)\right)$

Intuition: Splitting a class $x$ into two sibling classes $x_{1}$ and $x_{2}$

\begin{tabular}{lll}
\hline Exclusion changes $\left(\delta_{1}\right)$ & Pushout-1 & (type) \\
\hline$x$ rdf:type OWL:Class & delete node $x$ & $\left(m_{2}\right)$ \\
$x$ rdfs:subClassOf $z$ & delete edge $a_{1}$ & $\left(m_{2}\right)$ \\
$u_{1}$ rdfs:domain $z$ & delete edge $b_{1}$ & $\left(C_{d}\right)$ \\
$u_{2}$ rdfs:range $z$ & delete edge $b_{2}$ & $\left(C_{d}\right)$ \\
$i_{1}$ rdf:type $z$ & delete edge $b_{3}$ & $\left(C_{d}\right)$ \\
\hline Inclusion changes $\left(\delta_{2}\right)$ & Pushout-2 & $($ type $)$ \\
\hline$x_{1}$ rdf:type OWL:Class & add node $x_{1}$ & $\left(m_{3}\right)$ \\
$x_{1}$ rdfs:subClassOf $z$ & add edge $a_{3}$ & $\left(m_{3}\right)$ \\
$x_{2}$ rdf:type OWL:Class & add node $x_{2}$ & $\left(m_{3}\right)$ \\
$x_{2}$ rdfs:subClassOf $z$ & add edge $a_{4}$ & $\left(m_{3}\right)$ \\
\hline Inclusion changes $\left(\delta_{2}\right)$ & Pushout-3 & $($ type $)$ \\
\hline$u_{1}$ rdfs:domain $x_{1}, x_{2}$ & add edges $w_{1}, w_{2}$ & $\left(C_{d}^{\prime}\right)$ \\
$u_{2}$ rdfs:range $x_{1}, x_{2}$ & add edges $w_{3}, w_{4}$ & $\left(C_{d}^{\prime}\right)$ \\
$i_{1}$ rdf:type $x_{1}, x_{2}$ & add edges $w_{5}, w_{6}$ & $\left(C_{d}^{\prime}\right)$ \\
\hline Ontology conditions $(\phi)$ & Graph conditions $\left(C_{i}\right)$ & \\
\hline$x_{1}, x_{2} \notin O-x_{1}, x_{2} \in O^{\prime}$ & $x_{1}, x_{2} \notin G-x_{1}, x_{2} \in H$ & \\
$x \in O-x \notin O^{\prime}$ & $x \in G-x \notin H$ & \\
$z \in\left(O, O^{\prime}\right)$ & $z \in D$ & \\
$(x$ rdfs:subClassOf $z) \in O$ & $\operatorname{src}\left(a_{1}\right)=x \& \operatorname{tar}\left(a_{1}\right)=z$ in $G$ & \\
$\left(x_{1}\right.$ rdfs:subClassOf $\left.z\right) \in O^{\prime}$ & src $\left(a_{3}\right)=x_{1} \& \operatorname{tar}\left(a_{3}\right)=z$ in $H$ & \\
$\left(x_{2}\right.$ rdfs:subClassOf $\left.z\right) \in O^{\prime}$ & src $\left(a_{4}\right)=x_{2} \& \operatorname{tar}\left(a_{4}\right)=z$ in $H$ & \\
\hline & & \\
\hline
\end{tabular}

pushout 2 Similar to pushout 1 , first we identify the match of the reference gluing graph $K$ in the ontology gluing subgraph $D$ (i.e., $m_{2}: K \longrightarrow D$ ). Once a match is confirmed, production $r$ is applied to the ontology subgraph $D$ (through co-production $h$ ) resulting into an intermediate graph $H$ (i.e., $h=D \stackrel{r, m_{2}}{\Longrightarrow} H$ ). The co-production $h$ represents the addition of two concepts $x_{1}$ and $x_{2}$ in the ontology concept hierarchy. Thus, in Fig. 15, the nodes $x_{1}$ and $x_{2}$ are added to the gluing graph $D$ and are linked to available node $z$ through edges $a_{3}$ and $a_{4}$.

pushout 3 To ensure that the roles (cf. Fig. 16) of the deleted concept have been transferred to the newly added concepts, the effect of the dangling condition must be reversed. We call it inverse dangling condition $\left(C_{d}^{\prime}\right)$. Thus, all the edges that had been removed from the graph in pushout 1 (due to dangling condition) must be added back to the newly added concepts in pushout 3 .

In pushout 3 , the user can select different evolution strategies for inheriting the roles of the deleted concept by the newly added concept. For example, in case of the split change operation, the user can either (1) distribute the roles among

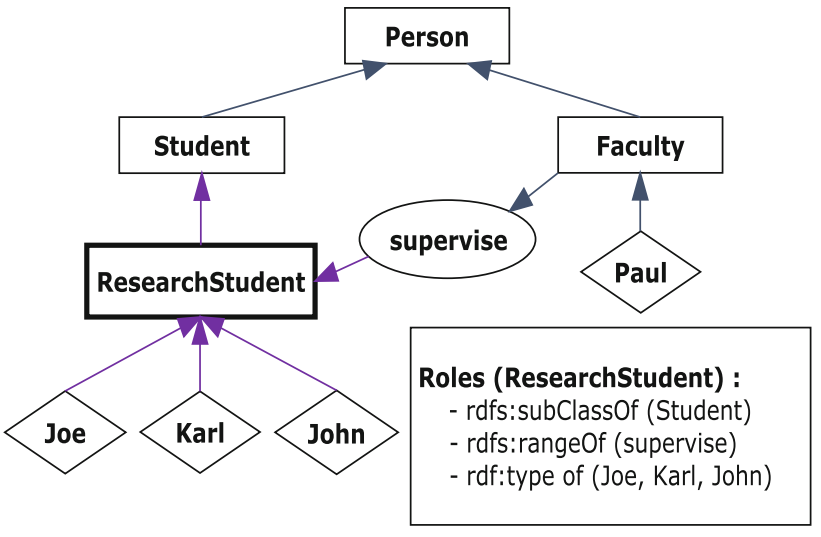

Fig. 16 Role of a concept ResearchStudent

the newly added concepts, (2) add the roles to both concepts or add the roles to one of the added concept $\left(i=H \stackrel{s, m_{3}}{\Longrightarrow} I\right)$. As in our running example, we chose option 2 , the nodes $u_{1}$, $u_{2}$ and $i_{1}$ are linked to the nodes $x_{1}$ and $x_{2}$.

\subsection{Detection of Composite Changes}

We operationalize the composite change detection in terms of graph matching. The TPO approach can be applied directly, if one preserves the different versions of the ontology. As we log the applied change operations, rather than the different versions of the ontology, we input the productions to the composite change detection algorithm, rather than the ontology and referenced ontology subgraphs. Thus, the input to the composite change detection algorithm is the change log graph (representing the applied atomic changes on the domain ontology) and the referenced composite change graph (representing the sequence of atomic changes to be identified) along with the specified conditions. Below, we describe some frequently used terms:

Session (s) The ontology change log graph is a collection of sessions $S$, where each session $s$ consists of the change log entries, from the time the domain ontology is loaded into the ontology editor, till the time it is closed. Thus, whenever an ontology is loaded into the editor, a new session starts and all the applied changes are recorded into the following session.

Graph node vs. TPO node One should differentiate between a graph node of a change log graph and a node given in a TPO diagram. In this paper, the term "graph node" represents a single ontology change log entry (i.e., representing a single atomic change) in a change log graph, where each graph node comprises a number of attributes such as target/auxiliary parameters, operation, element, session id, etc. (cf. Fig. 11). The term "TPO node", represents an ontology entity (i.e., concept, property, individual, etc.) in the TPO Fig. 15.

Role The term "role" refers to the usage of an ontology entity in a specific ontology version. For example, in Fig. 16, 
Table 2 List of composite change patterns and their definitions

\begin{tabular}{ll}
\hline Composite change & Description \\
\hline Split concept $\left(x,\left(x_{1}, x_{2}\right)\right)$ & Split a concept $x$ into two newly created sibling concepts $x_{1}$ and $x_{2}$ \\
Merge concept $\left(\left(x_{1}, x_{2}\right), x\right)$ & Merge two existing concepts $x_{1}$ and $x_{2}$ into one newly created \\
concept $x$ & and cumulate all roles of $x_{1}$ and $x_{2}$ into $x$ \\
Pull up concept $\left(x, x_{1}\right)$ & Pull concept $x$ up in its class hierarchy \\
& and attach it to all parents of its previous parent $x_{1}$ \\
Pull up concept $(x)$ & Pull concept $x$ up in its class hierarchy \\
& and attach it to all parents of all its previous parents \\
Pull down concept $\left(x, x_{1}\right)$ & Pull concept $x$ down in its class hierarchy \\
& and attach it as a child to its previous sibling concept $x_{1}$ \\
Pull down concept $(x)$ & Pull concept $x$ down in its class hierarchy \\
& and attach it as a child to all its previous sibling concepts \\
Move concept $\left(x, x_{1}\right)$ & Detach concept $x$ from its previous superclass \\
& and attach it as a subclass to a concept $x_{1}$ \\
Group concepts $\left(x,\left(x_{1}, x_{2}\right)\right)$ & Create a common parent concept $x$ for sibling concepts $x_{1}$ and $x_{2}$ \\
& and transfer the common properties to it \\
Add generalisation concept & Add a new concept $x$ between $x_{1}$ and all its super classes \\
$\left(x, x_{1}\right)$ & Add a new concept $x$ between $x_{1}$ and all its subclasses \\
Add specialization concept & Pull a property $p$ up in the class hierarchy \\
$\left(x, x_{1}\right)$ & and attach it to the superclass $x_{2}$ of its previous domain/range concept $x_{1}$ \\
Pull up property $\left(p, x_{1}, x_{2}\right)$ & Pull a property $p$ down in the class hierarchy \\
Pull down property $\left(p, x_{1}, x_{2}\right)$ & and attach it to the subclass $x_{2}$ of its previous domain/range concept $x_{1}$ \\
\hline &
\end{tabular}

the concept ResearchStudent has five roles i.e, subclass of concept Student, range of object property isSupervisorOf and the type of individuals Joe, Karl and John.

\subsubsection{Algorithms for Composite Change Detection}

There is no agreed standard set of composite change operations that one could be based on. It is obvious (and also mentioned in previous research $[44,27])$ that one can combine different atomic level change operations to construct new composite changes. Thus, providing an exhaustive list of composite change operations is not feasible. In our current work, we select the composite change patterns and their definitions from Stojanovic [44] and they are given in Table 2.

The basic idea of the composite change detection algorithm is to iterate over each session of the change log graph and find the location from where an applied composite change may start. Pass the identified location's session node $n_{g}$ and the reference graph $G_{r}$ to a function that extracts the complete sequence of nodes (starting from $n_{g}$ ) that maps completely to $G_{r}$. In the mapping step, ensure that the correlations among the parameters of the identified change operations are satisfied.

\subsubsection{Description of Algorithm}

The complexity of the presented algorithm is linear $O(n)$. The composite change detection algorithm is given in List- ings 1.1 and 1.2, where Listing 1.1 describes the main algorithm and Listing 1.2 presents algorithm for one of the function (method). Below, we describe the algorithm in form of steps (and sub-steps):

\section{Listing 1.1:}

Step A: The algorithm takes the change $\log$ graph $G$ and reference graph $G_{r}$ as an input and group the graph nodes into a set of sessions (lines 1-2).

Step B: Once we have the session set $S$, the algorithm iterates over each session $s$ (lines 3-18).

Step B.1: Within each iteration over session $s$, first we get the range of the session by extracting the node ids of the first and the last node of the session. The parameter current Id (representing the id of the currently visited graph node) is initialized with the first node id (lines 4-6).

Step B.2: We iterate over the graph nodes of session, until the id of the currently visited node is less than the id of the last node of the session (lines 7-17).

Step B.2.1: In each iteration, we extract the first node $n_{r}$ from the reference graph $G-r$ and identify a matching node to $n_{r}$ from the log session $s$ (lines 8-9).

Step B.2.2: If no matching node is identified from the session, the algorithm goes back to step 3 to select the next session from the session set (lines 10-11).

Step B.2.3: If a matching node is identified from the session, the algorithm passes the matched node $n_{g}$, reference composite change graph $G_{r}$ and the session $s$ to a method, 
i.e., matchPattern(), that identifies the complete composite change sequence (line 13).

Step B.2.4: The method matchPattern() returns a list of change operations (representing a detected composite change operation) that is passed as an output of the algorithm or returns a null value (representing that composite change was not identified at particular location of the session) (lines 1316).

\section{Listing 1.2:}

Step A: First, we save the passed graph node $n_{g}$ in an extendable list (line 1).

Step B: We iterate over the session $s$, as long as the complete composite change reference graph is not identified (lines 2-12).

Step B2.1: In each iteration, we select the subsequent nodes of the reference graph $G_{r}$ and the session $s$ (lines 3-4).

Step B2.2: We match the selected nodes. If the nodes are matched and the correlations are satisfied, the selected node $n_{g}$ is added into the list and the next subsequent node of the session $s$ is selected as a current node (lines 5-7).

\section{Listing 1.1: Composite Change Detection Algorithm}

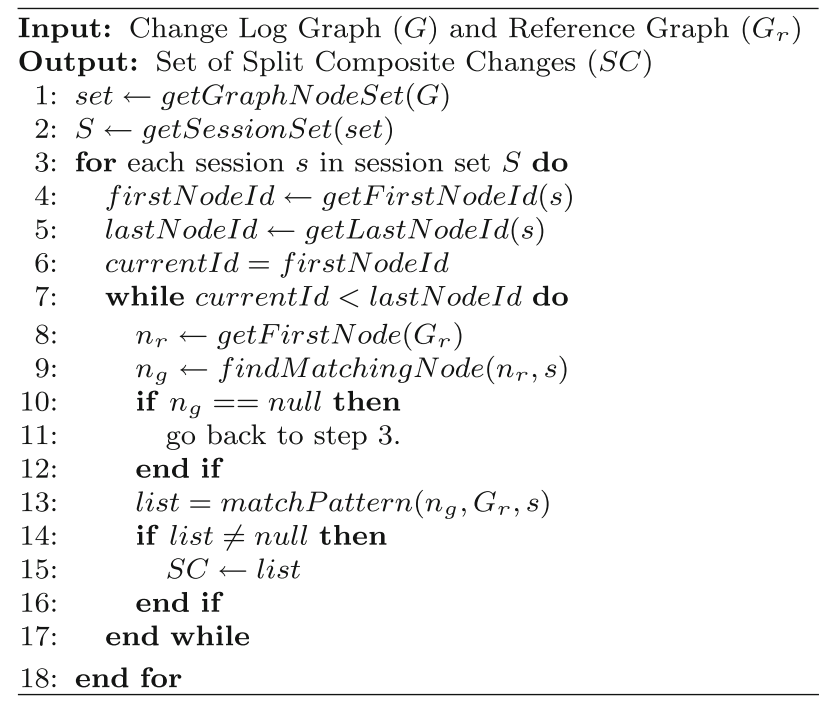

\section{Listing 1.2: Method: matchPattern()}

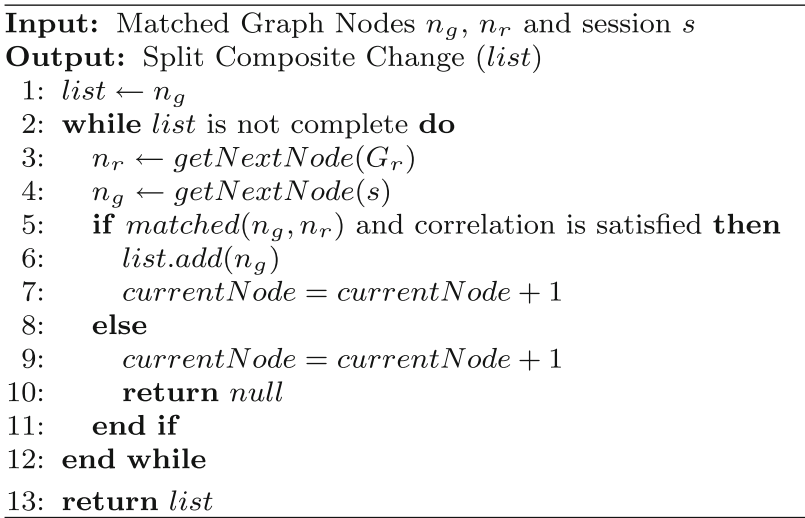

Step B2.3: If the nodes do not match (in above step B2.2), the next subsequent node of the session $s$ is selected as a current node (lines 5-7) and the algorithm goes back to Listing 1.1 (from where this method was called) with a null value returned.

\section{Change Patterns Discovery}

Graph-based formalisation (discussed in Sect. 5.3) allows us to identify and classify frequent changes that occur in domain ontologies over a period of time. Initially, we analyzed the change log graph manually and observed that combinations of change operations occur repeatedly during the evolution of ontologies. We identified these as frequent recurring change patterns that can be reused.

\subsection{Analysis of Change Log Graph}

While patterns are sometimes used in their exact form, users often use different orderings of change operations to perform the same (semantically equivalent) changes at different times. To capture semantically equivalent, but operationally different patterns, more flexibility is needed. We introduce a metric, called sequence gap or generally $n$-distance, that captures a node gap between two adjacent graph nodes in a sequence [23]. It refers to the distance between two adjacent graph nodes in a change log graph. This helps us to define a more flexible pattern notion. We merge different types of patterns into two basic subdivisions, i.e.

\section{- Ordered Change Patterns $(O P)$ \\ - Unordered Change Patterns (UP)}

The instances of the ordered change patterns comprise change operations in exact same sequential order from a change log graph. Thus, such complete (OCP) or partial (OPP) patterns may have only a positive node distance value, starting from zero to a user given threshold $(x)$. The instances of unordered change patterns comprise change operations which may or may not be in the exact same sequential order in a change log graph. These complete (UCP) or partial (UPP) patterns may have a node distance that ranges from a negative node distance value $(-x)$ to a positive node distance value $(x)$. Completeness means that all pattern nodes are used in the concrete graph; partiality refers to a subset of nodes. For the remainder, we focus on complete change patterns, but we discuss the relevance of partial change patterns in our conclusions.

Metrics: We consider identifying recurring sequenced change operations from a change log as a problem of recognition of frequent patterns in a graph. First we describe the key metrics.

Definition 1 Pattern Support: The pattern support of a pattern $p$ is the number of occurrences of such a pattern in 
the change log graph $G$. Pattern support is denoted by $\sup (p)$. The minimum number of occurrences required for a sequence $s$ in change $\log$ graph $G$ to qualify as a change pattern $p$ is the minimum pattern support, denoted by min_ $\sup (p)$.

Definition 2 Pattern Length: The pattern length of a pattern $p$ is the number of change operations in it, denoted by $\operatorname{len}(p)$. The minimum length required for a sequence $s$ in a change $\log$ graph $G$ to qualify as a member of a candidate pattern set is the minimum pattern length, denoted by min_ $\operatorname{len}(p)$.

Definition 3 Candidate Change Pattern Sequence: For a given $\mathrm{ACL}=<a c_{1}, a c_{2}, a c_{3} \cdots a c_{n}>$, a candidate pattern sequence $c s$ is a sequence $<a c_{p 1}, a c_{p 2}, a c_{p 3} \cdots a c_{p k}>$ with

- $a c_{p i} \in A C L$ for $i=1,2 \cdots k$ and

- if $\operatorname{pos}\left(a c_{p i}\right)<\operatorname{pos}\left(a c_{p j}\right)$ in $c s$, then $\operatorname{pos}\left(a c_{p i}\right) \leq$ $\operatorname{pos}\left(a c_{p j}\right)$ in $A C L \ldots$ for all $i=1 \cdots k-1$ and $j=2 \cdots k$.

Definition 4 Change Pattern Sequence: A candidate change pattern sequence $c s$ is a discovered change pattern $p$ if

- $l e n(c s) \geq$ min_len $(p)$.

i.e., the length of the candidate pattern sequence $c s$ is equal to or greater than the threshold value set by the minimum pattern length.

- $\sup (c s) \geq$ min_sup $(p)$.

i.e., the support for the candidate pattern sequence $c s$ in a change $\log$ graph $G$ is above the threshold value of minimum pattern support.

Definition 5 Definition 5 - Ordered Change Pattern: Let a change pattern $p=<s_{1}, s_{2} \cdots s_{d}>$ be a set consisting of a candidate change pattern sequence $c s\left(c s=s_{1}\right)$ and the change pattern support sequences $\left(s_{2}, s_{3} \cdots s_{d}\right)$ for $c s$. The change pattern $p$ is an ordered change pattern $(O P)$ with

- $s_{i}=<a c_{i 1}, a c_{i 2} \cdots a c_{i n}>\in p$ for $i=1 \cdots d$

- if $\operatorname{pos}\left(a c_{i x}\right)<\operatorname{pos}\left(a c_{i y}\right)$ in $s_{i}$ then $\operatorname{pos}\left(a c_{i x}\right) \leq \operatorname{pos}\left(a c_{i y}\right)$ in $A C L$ for all $x=1 \cdots n-1, y=2 \cdots n$.

Definition 6 Unordered Change Pattern: Let $u$ and $v$ be the minimum and maximum positions in an identified change pattern sequence $s_{i}$, respectively. The change pattern $p$ $\left(p=<s_{1}, s_{2} \cdots s_{d}>\right)$ is an unordered change pattern $(U P)$ with

- $s_{i}=<a c_{i 1}, a c_{i 2} \cdots a c_{i n}>\in p$ for $i=1 \cdots d$

- if $u=$ min_pos $\left(a c_{i 1}, a c_{i 2} \cdots a c_{i n}\right)$ in $s_{i}$ and $v=$ max_pos $\left(a c_{i 1}, a c_{i 2} \cdots a c_{i n}\right)$ in $s_{i}$, then $u \leq \operatorname{pos}\left(a c_{i}\right)$ $\leq v$ in $A C L$.

\subsection{Change Patterns Discovery Algorithms}

The identification of domain-specific change patterns is operationalised in the form of discovery algorithms. The section is divided into two parts, i.e. algorithms for searching ordered complete change patterns (OCP) and algorithms for searching unordered complete change patterns (UCP). The inputs to the pattern discovery algorithms comprise the graph $G$ representing change log triples, the minimum pattern support min_sup, the minimum pattern length min_len and the maximum node-distance $x$. Before we describe each algorithm, we introduce some concepts.

- Target entity, primary/auxiliary context of change: The target entity is the ontology entity to which the change is applied; primary/auxiliary context refers to entities which are directly/indirectly affected by such a change.

- Candidate node $(c n)$ : A candidate node $c n$ is a graph node selected at the start of the node iteration process (discussed later). Each graph node will act as a candidate node $c n$ in one iteration each of the algorithm.

- Candidate sequence (cs): The candidate sequence $c s$ is the context-aware set of graph nodes starting from a particular candidate node $\mathrm{cn}$.

- Discovered node $(d n)$ : The discovered node $d n$ is a graph node that matches the candidate node $c n$ (in a particular iteration) in terms of its operation, element and type of context. $D N$ refers to the set of discovered nodes.

- Discovered sequence ( $d s): d s$ is the context-aware set of graph nodes starting from a discovered node $d n$ that matches candidate sequence $c s$ (in an iteration). $D S$ refers to the set of discovered node sequences.

\subsubsection{OCP Discovery Algorithm}

To discover ordered complete change patterns (OCP), the identified sequences are of the same length and contain change operations in the exact same chronological order. The basic idea of the algorithm is to iterate over the graph nodes, generate the candidate sequence starting from a particular graph node and search the similar sequences within the graph $\mathrm{G}$. The $O C P$ algorithm is defined in Listings 2.1$2.2 \%$ and explained below. The algorithm iterates over each graph node and selects it as a candidate node $\left(c n_{k}\right)$, where $k$ refers to the identification key of the node. Once the candidate node is selected, an iterative process of expansion of candidate node $c n_{k}$ to its adjacent nodes $c n_{k++}$ starts and continues until more expansion is not possible (i.e. adjacent node do not share the same target entity). If the target entity of the adjacent node is matched with the target entity of the candidate node, it is taken as the next node of the candidate sequence $c s$. If the target entity does not match, an iterative 
Listing 2.1: OCP Discovery Algorithm

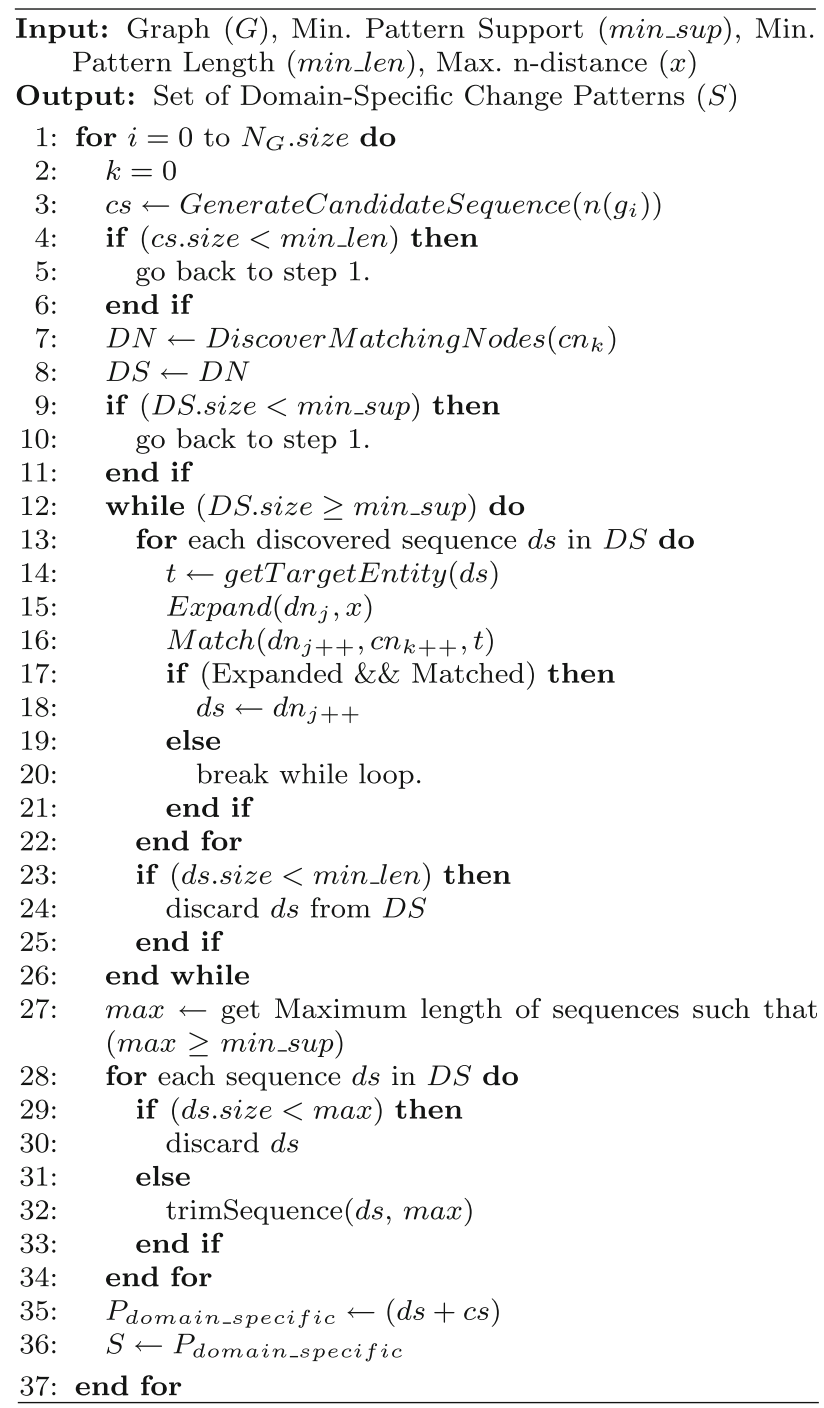

process will start to find the next node whose target entity matches the target entity of the candidate node. The iteration continues based on the user threshold $x$, i.e. the allowed gap between two adjacent graph nodes of a pattern (n-distance).

Once the candidate sequence is constructed and is above the threshold value for the minimum pattern length, the next step is to search for the matching nodes (i.e. discovered nodes $d n$ ) of the same type as the candidate node $c n_{k}$. If the number of discovered nodes is above the threshold value (minimum pattern support), the next step is to expand the discovered nodes and match them to parallel candidate nodes. Each discovered node is expanded one after another. Similar to the expansion of candidate nodes, the identification of the next node of a discovered sequence $d s$ is an iterative process (depending on $x$ ).

The expansion of a discovered node $d n$ stops if either more expansions of that node are not possible or the expansion has
Listing 2.2: GenerateCandidateSequence()

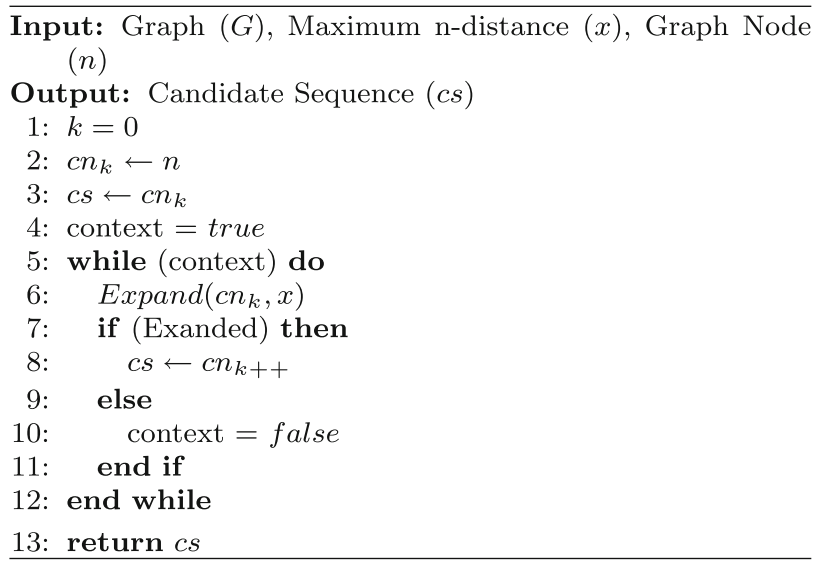

reached the size of the candidate sequence (i.e. the length of $d s$ is equal to the length of $c s$ ). At the end of the expansion of a discovered sequence, if the length of an expanded discovered sequence is less than the threshold value of the minimum pattern length, it must be discarded from the set of discovered sequences.

Once the expansion of discovered nodes is finished, in order to identify the change patterns of greater size, the next step is to find the maximum length of the sequences ( $\max$ ) such that the value of $\max$ is greater than or equal to threshold value of the minimum pattern length and the number of identified sequences is greater than or equal to the threshold value of minimum pattern support. Sequences whose length is less than the value max are discarded from the set of discovered sequences. Those discovered sequences whose length is greater than $\max$ are truncated to size $\max$.

As a last step, the candidate sequence along with the discovered sequences is saved as a domain-specific change pattern in the result list $S$ and the algorithm goes back to step 1 and selects the next graph node as a candidate node.

\subsubsection{UCP Discovery Algorithm}

A collection of change operations is not always executed in same chronological order, even if the result is the same. As then the change operations in a sequence can be reordered, the aim is to discover unordered complete change patterns by modifying the node search space in each iteration. The pseudocode of the UCP algorithm is given in Listings 3.13.2.

Like OCP, UCP iterates over each graph node and selects it as a candidate node $\left(c n_{k}\right)$. An iteration is used to construct a candidate sequence $c s$ by expanding candidate node $c n_{k}$ to its subsequent context-matching nodes $c n_{k++}$. The next step identifies the discovered nodes $d n$ and adds them as first member of the discovered sequence set $D S$. There are two differences in the expansion of discovered sequences in UCP 


\section{Listing 3.1: UCP Discovery Algorithm}

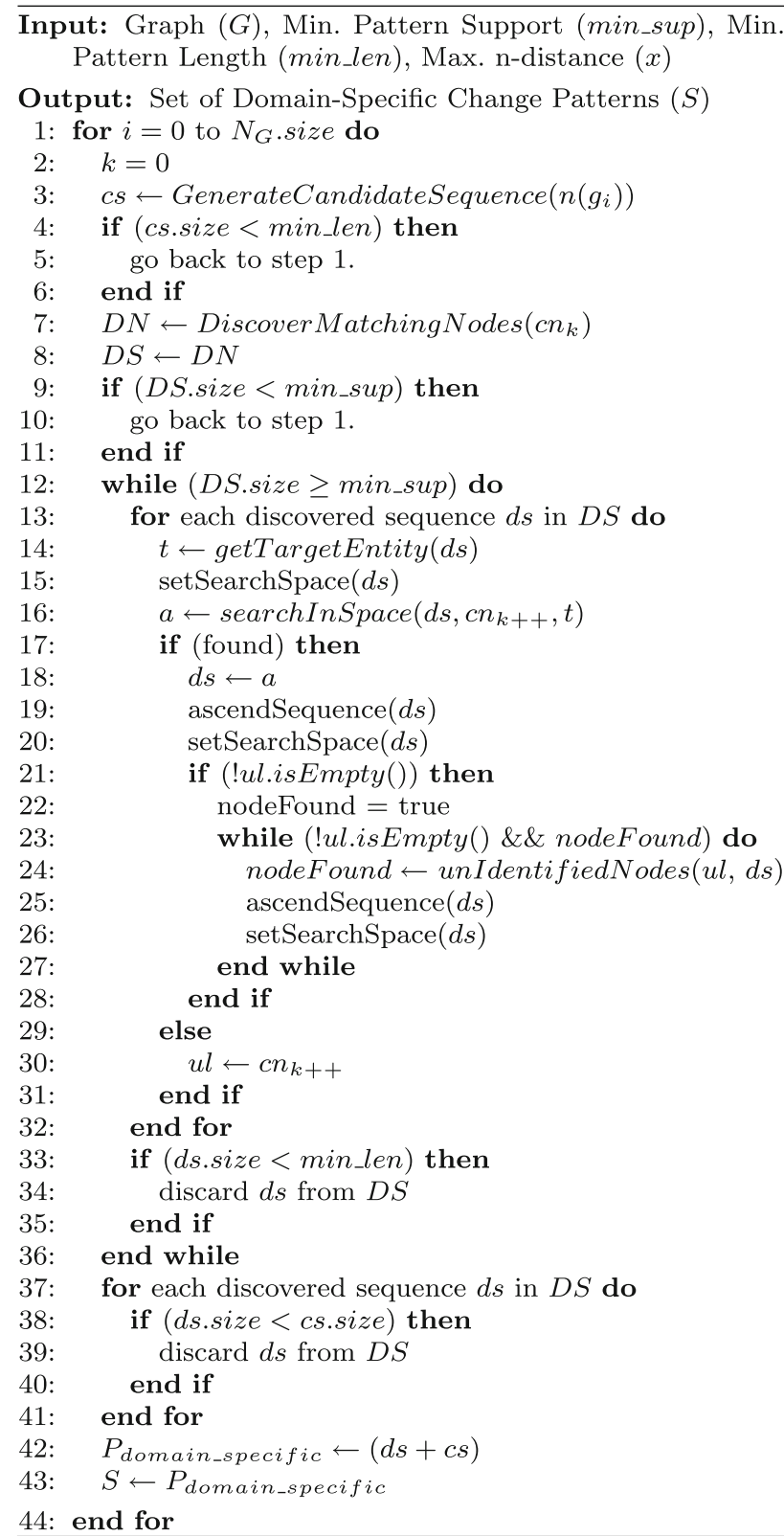

and OCP. Firstly, the search space in which the mapping graph node will searched and, secondly, the introduction of an unidentified-nodes list $(u l)$ which records the unidentified nodes of a candidate sequence.

Before the expansion process on any discovered node starts, the search space (i.e. range of graph nodes in which node will be searched) has to be set. It is described using two integer variables start_range $\left(r_{s}\right)$ and end_range $\left(r_{e}\right)$, where $r_{s}$ and $r_{e}$ represent the node ids of the start and end graph nodes of search space. The search space can be given as $r_{s}=\min (i d)-x-1$ and $r_{e}=\max (i d)+x+1$.

Values $\min (i d)$ and $\max (i d)$ are the minimum and maximum $i d$ values of the graph nodes in the discovered sequence
Listing 3.2: setSearchSpace()

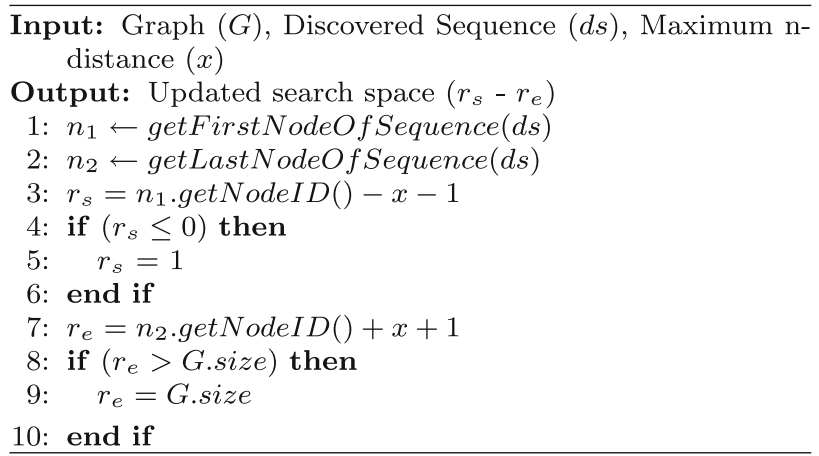

$d s$ in a particular iteration. New values of $r_{s}$ and $r_{e}$ are calculated at the start of each iteration of the discovered node expansion process. For example, given the gap constraint $(x=1)$ and a discovered sequence $d s$ that contains two graph nodes $d s=\left\{n_{9}, n_{11}\right\}$ in a particular iteration, the search space (in which the next discovered node will be searched) is $n_{7}-n_{13}$. As the algorithm scans the whole graph only once (i.e. in step 7 of algorithm 4.3 to get the discovered node set) and narrows the search space later, the search space defining technique improves the performance of the algorithm.

The unidentified nodes list $(u l)$ records all candidate nodes that are not matched in the $d s$ expansion process. If a new node is added to a discovered sequence, the sequence will be converted into ascending form (based on their $i d$ values) and the search space is reset. If there is no match and $d s$ is not expanded, the respective candidate node is added to $u l$. Once the discovered sequence $d s$ is expanded, an iteration is applied on $u l$ to search the unidentified nodes in the updated search space. If an unidentified candidate node is matched to a discovered node in the updated search space, the node is added to the discovered sequence and removed from the unidentified node list. Based on the modified discovered sequence, the values of $r_{s}$ and $r_{e}$ are recalculated.

At the end of the expansion of a discovered sequence, if the length of an expanded discovered sequence is less than the minimum pattern length threshold, it must be discarded from the set of discovered sequences. Then, all discovered sequences whose length is less than the length of a candidate sequence are discarded. As a last step, the candidate sequence along with discovered sequences is saved as a change pattern in result list $S$ and the algorithm goes back to step 1 and selects the next graph node as a candidate.

\section{Experimental Results and Evaluation}

The main concern in evaluating the layered change operator and $\log$ framework is its practical validity and the adequacy. 
Table 3 List of change operations and their type

\begin{tabular}{lll}
\hline No. & Type & Change \\
\hline 1 & Atomic & Add class (Lecturer), Add subClassOf (Lecturer, Faculty) \\
2 & Atomic & Add individual (John), Add classAssertion (John, UGStudent) \\
3 & Composite & $\begin{array}{l}\text { Split class (ResearchStudent, (MSByResearchStudent, } \\
\text { PhDResearchStudent)), Strategy: Split the Roles }\end{array}$ \\
& & Merge classes ((MSByResearchStudent, PhDResearchStudent), \\
4 & Composite & ResearchStudent), Strategy: Aggregate all roles \\
& & Copy class (ResearchStudent, ResearchIntern, Researcher) \\
5 & Composite & Split class (ResearchStudent, (MSByResearchStudent, \\
6 & Composite & PhDResearchStudent)), Strategy: Attach to both classes \\
& & PhD Student Registration (Tylor Kane, 58106382, \\
7 & Domain-specific & tylor@ computing.dcu.ie, Joe Morris, Computing, CNGL, Irish) \\
& & Add New University Event (AICS 2012, ResearchEvent, 17 Sep 2012, 19 \\
8 & Domain-specific & Sep 2012, 23rd Irish Conference on Artificial Intelligence and Cognitive \\
& & Science, Ray Walshe, aisc2012@ comuting.dcu.ie, +353-1 700 597) \\
\hline
\end{tabular}

How useful the proposed solution is and how effectively it solves the problems faced in the real world. In terms of change pattern identification algorithms, the effectiveness of the algorithms in terms of correctness and completeness are the key factors. Empirical case studies and lab-based experiments, in a controlled environment, can be used to evaluate any system and to accept or reject the effectiveness of methods, techniques or tools [9]. We selected an empirical case study and controlled experiments as our evaluation strategies. In Section 8.1, the user-based evaluation of the proposed change operator framework is given. In Section 8.2, the results and evaluation of our controlled experiments, being done to identify the composite change patterns from an ACL, are given. The change pattern discovery algorithms have been evaluated based on the experiments in a few domain ontologies. Results are given in Section 8.3.

\subsection{Layered Change Operator Framework}

Different levels of change patterns emerge by clustering the empirically observed frequent changes in the domain ontology. These change patterns are useful for the ontology engineers to modify domain ontologies more easily and more correctly.

\subsubsection{Evaluation}

We involved few ontology engineers for evaluating the framework in terms of its change operational cost. The change operational cost has been evaluated in two ways, i.e., in terms of the number of steps to be performed and the time required performing the specified steps. To do so, we selected eight different ontology change operations, two from the atomic level (level one), four from the composite level (level

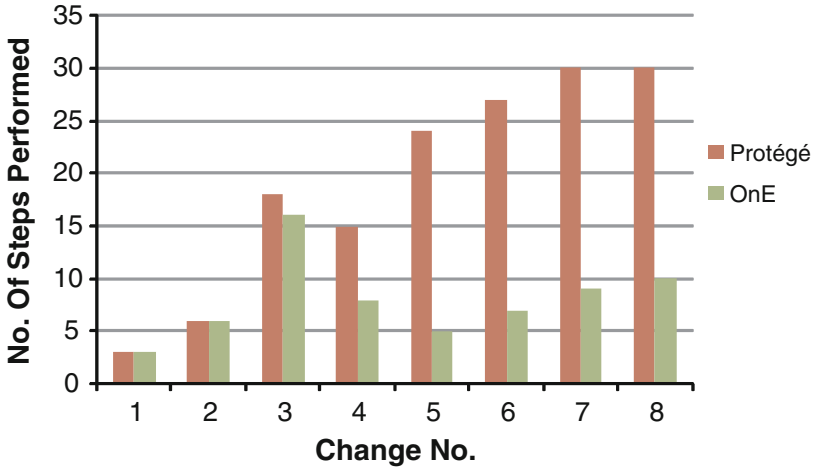

Fig. 17 Protege vs. OnE: number of steps performed

two) and two from the domain-specific level (level three) Table 3.

Operational cost in terms of number of steps We evaluated the framework on the basis of the number of steps required to perform the specific changes given in Table 3. To do so, we make use of our Ontology Editor (OnE) and widely used Protégé framework. Results are given in Fig. 17 in the form of bar chart. It is evident that in case of atomic level change operations, both frameworks require the same number of steps to be performed. However, usage of evolution strategies [25] and pattern-driven data entry forms (for performing higher-level change operations) significantly reduces the evolution effort in terms of the number of required steps. For example, in case of composite change operation Merge classes (change 4), user need to take eight (8) steps (in OnE) in comparison to fifteen (15) changes in Protégé. The biggest difference was seen in case of the Split class composite change where the selected strategy was to join the roles to both the newly added classes (change 6). The result is fairly understandable as in case of Protégé, users need to attach each role one after the other and hence increase the number of required steps. The more roles to be attached, the more the steps it requires. 
Table 4 Comparison between OnE and Protégé (min:sec)

\begin{tabular}{llllllll}
\hline \multirow{2}{*}{ No. } & \multicolumn{2}{l}{ Protégé } & & & & \multicolumn{2}{l}{ OnE } \\
\cline { 8 - 9 } \cline { 7 - 8 } & Min & Max & Avg & & Min & Max & Avg \\
\hline 1 & $0: 03$ & $0: 12$ & $0: 06$ & & $0: 04$ & $0: 10$ & $0: 06$ \\
2 & $0: 11$ & $0: 34$ & $0: 21$ & & $0: 07$ & $0: 24$ & $0: 17$ \\
3 & $0: 55$ & $3: 21$ & $1: 53$ & & $0: 22$ & $2: 08$ & $0: 57$ \\
4 & $0: 35$ & $1: 18$ & $1: 05$ & & $0: 11$ & $0: 39$ & $0: 20$ \\
5 & $0: 37$ & $1: 55$ & $1: 09$ & & $0: 07$ & $0: 21$ & $0: 12$ \\
6 & $1: 03$ & $1: 42$ & $1: 26$ & & $0: 09$ & $0: 39$ & $0: 19$ \\
7 & $0: 51$ & $2: 34$ & $1: 40$ & & $0: 17$ & $1: 40$ & $0: 57$ \\
8 & $1: 26$ & $2: 59$ & $2: 00$ & & $0: 31$ & $1: 52$ & $1: 08$ \\
\hline
\end{tabular}

On the other hand, in case of OnE, users only need to select the appropriate evolution strategy and all roles will automatically be attached to the newly added split classes. Hence, increase or decrease of roles does not have any effect on the number of required steps.

Operational cost in terms of time We evaluated the framework based on the time required to perform the different level of change operations. We compared the time taken by the ontology engineers (minimum, maximum and average) for performing the changes in both ontology editing frameworks. The performance comparison is given in Table 4 . Learning affects on the performance of different users have been considered and factored in our controlled experiment. We observed that on average the time occupied by the two ontology editing frameworks to perform an ontology change using atomic change operators is in a similar range. However, the usage of higher level change operators and the evolution strategies had a reasonable impact on the required time (change nos. 3-6). For example, in case of performing Merge classes (change 4) in Protégé, user need to attach each role one after the other. As we mentioned earlier, the more roles, the more time it is going to take. On the other hand, selecting evolution strategy "Aggregate all roles" reduced the time required for attaching all the roles. Similarly, in case of split class (change 6), by selecting evolution strategy "Attach to both classes" the user did not need to attach roles to the split classes one after the other.

\subsubsection{Practical Benefits}

The ontology change patterns can be used as data entry forms in an existing ontology editing toolkit. These (generic/ domain-specific) change patterns (along with the different defined evolution strategies) are useful for the ontology engineers to modify domain ontologies more easily and more correctly. The consistency issues during ontology evolution can be resolved using evolution strategies at each layer of change operator framework. As discussed above, the usage of

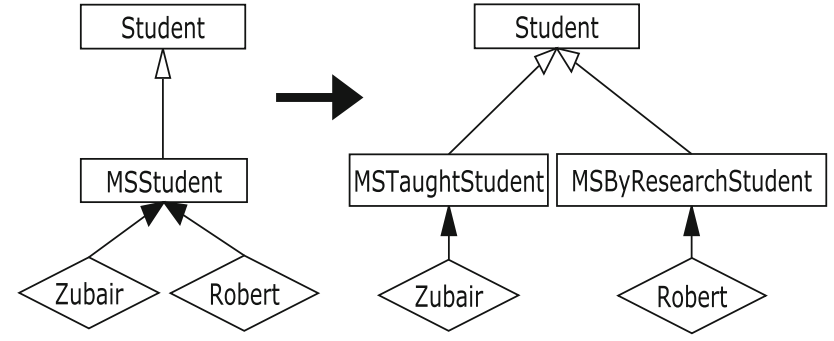

Fig. 18 Example of an identified composite change

pattern-driven data entry forms (for performing higher-level change operations) significantly reduces the evolution effort in terms of time and manual effort. Furthermore, the change pattern data entry forms also make the evolution process intuitive and simple for a non-expert.

\subsection{Composite Change Detection Algorithms}

Detection of composite changes not only helps in understanding the evolution of domain ontologies, but also reduces the effort required in terms of time and consistency management. Based on the identified composite changes, more appropriate (composite level) strategies can be employed to keep the validity and consistency.

In this section, first we illustrate a few examples of the identified composite changes. Second, we evaluate the composite change pattern detection algorithms based on the controlled experiments and their comparison with a manual approach. At the end, we describe the learnt lessons (methodology) from the controlled experiments and their results.

\subsubsection{Illustration of Results}

Two examples from the identified composite changes are given in Figs. 18 and 19.

The example given in Fig. 18 represents an identified split change, where, "Distribute the roles" was the selected evolution strategy. In a previous version of the ontology $V_{1}$, concept Student was classified into MSStudent, PhDStudent and UGStudent. Thus, all the master's students (OWL:Individual), whether taught or research-based, were direct instances of concept MSStudent. In subsequent version of ontology $V_{2}$, in order to distinguish between researchbased and course-based students of master's degree, the concept MSStudent is splitted into two sibling concepts (i.e., MSByResearchStudent and MSTaughtStudent). Based on the selected evolution strategy, the direct instances of the deleted concept MSStudent are distributed among the newly added concepts.

The example given in Fig. 19 represents an identified pull up property change on concept PhDStudent; where concepts MSByResearchStudent and PhDStudent were direct 


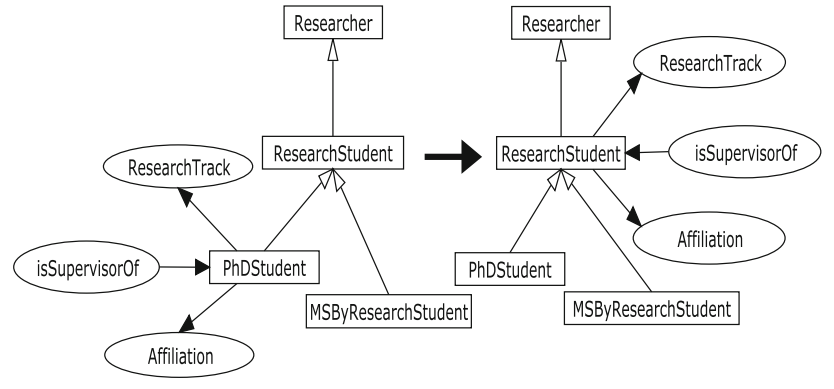

Fig. 19 Identified composite change: "Pull Up Property"

subclasses of concept student. In a previous version of the ontology $V_{1}$, the concepts MSByResearchStudent and PhDStudent are grouped under the concept ResearchStudent. In this regard, the next step was to pull up the properties to the common superclass ResearchStudent in the subsequent version $V_{2}$. We identified the composite changes such as "Pull up property (ResearchTrack)", "Pull up property (isSupervisorOf)", etc.

As we mentioned earlier, the semantics of any change must be captured at a higher level. Knowing that domain/range of a property $p$ has been changed from one concept to another, let us say from $x$ to $z$, certainly exhibits that the individuals of concept $x$ and of any other concept in its subclass hierarchy, who instantiate property $p$, are not valid anymore. However, knowing that the domain/range of a property $p$ has been generalised from $x$ to $z$ (vz. the concept $x$ is a subclass of concept $z$ ), assures that the validity of any of the individual is not violated. Similarly, in case of specialization of domain/range of a property $p$ from a parent concept $a$ to its (direct) child concept $b$ assures that the only those individuals of concept $a$ who instantiate property $p$, are not valid anymore. All other individuals of concept $a$ and others in the subclass concept hierarchy of $a$ (vz. the concept hierarchy starts from concept a) are still valid.

\subsubsection{Evaluation}

We evaluate the algorithms based on their completeness and correctness. In terms of completeness, the algorithms written to identify the composite change patterns should capture all types of available composite changes from the change $\log$. In terms of correctness, there should not exist any false identified composite change in the result list. It is obvious that an automated solution to identify change patterns from the change log will be faster than the manual identification of change pattern; thus, reduction in time consumption has not been considered as an evaluation criterion, but as a benefit.

We measured the completeness and correctness of our composite change pattern detection algorithms by comparing their results with the manual approach. In this regard,
Table 5 Comparison between manual and automated composite change pattern detection

\begin{tabular}{lcc}
\hline & Manual & Automated \\
\hline Change log size & 120 atomic changes \\
Identified change patterns & 10 & 11 \\
Candidate change patterns & 1 & 1 \\
Complete change patterns & 9 & 10 \\
False change patterns & 0 & 0 \\
Missed change patterns & 1 & 0 \\
Time taken & 55 min & $<1 \mathrm{~s}$ \\
\hline
\end{tabular}

we gathered a small group of ontology engineers together and gave them a brief description about the domain (i.e., university administration), the composite changes and their definitions. We performed the evaluation in two steps:

- Step 1: We distributed among them the first five sessions of the ontology change log and asked them to identify the discussed composite changes from these change log sessions (Completeness). To perform the evaluation on a small scale, we selected only six types of composite change patterns (i.e., split concept, add specialize concept, group concepts, add interior concept, pull up property and pull down property) and a small size of ACL (i.e., 120 atomic ontology changes).

- Step 2: At the end of step 1, we gave them the results of our controlled experiments (i.e., results of the automated approach) and asked them to testify whether the detected composite changes are valid (Correctness).

Table 5 gives the details of the comparison between manual and automated detection of composite change patterns. Here in the table, the term "candidate" change pattern refers to the identified change patterns that as a whole or part of them can be acknowledged as a composite change pattern. The candidate change patterns identified through the manual or automated approach need to be reviewed again by an expert ontology engineer, before confirming them as correctly identified composite change patterns.

The ontology engineers were able to identify ten composite changes in comparison to the automated approach where the number of detected composite changes was 11 . It has been observed the ontology engineers were able to identify almost all the composite changes, but the main difference lies in two cases, i.e., the time taken to identify these changes (from such a small change log) and the missing of composite change patterns having a positive $n$-distance (cf. Sect. 7.1) .

- Ontology engineers took almost $1 \mathrm{~h}$ to go through 120 atomic ontology changes and to identify correct change 
patterns. This result shows that identifying composite change patterns manually, on a small scale change log, is possible but yet at a very high cost of time consumption (as the ontology engineers took almost $30 \mathrm{~s}$ to go through and relate a single atomic ontology change with other changes). In real world case scenario, the ontology change logs are of larger size and an automated solution is a necessity there. As the size of change log increases, the time required to identify composite change pattern manually will increase intensively and using some automated approach is inevitable.

- The manual approach missed the identification of an "add specialise concept" composite change pattern, during to the availability of few extra change operations in between the change operations of the composite change. This shows that the manual identification of a composite change pattern, where all the atomic change operations are in a sequence with zero n-distance between them, is relatively easier in comparison to the identification of a composite change pattern, where atomic change operations have some positive node distance between them.

\subsection{Change Pattern Discovery Algorithms}

When ontologies are large and in a continuous process of change, our pattern discovery algorithms can automatically detect change patterns. Such patterns are based on operations that have been used frequently. This reduces the effort required in terms of time consumption and consistency management.

Earlier, we presented pattern-based ontology change operators in section 4 and motivated the benefits of pattern-based change management where patterns are usually domainspecific compositions of change operators. Our work here can be utilized to determine these patterns and make them available for reuse.

- The key concern is the identification of frequent change patterns from change logs. In the first place, these are frequent operator combinations and can result in generic patterns. However, our observation is that many of these are domain-specific, as the example below will illustrate.

- This can be extended to identify semantically equivalent changes in the form of a pattern. For instance, a reordering of semantically equivalent operations needs to be recognised by the algorithms.

\subsubsection{Illustration of Results}

Two examples from discovered change pattern sequences, one from each level, i.e. ABox-based change patterns and TBox-based change patterns, are given in Tables 6 and 7 .
Table 6 ABox-based change pattern (extracted from University Ontology)

Table 7 TBox-based change pattern (extracted from Software Ontology)

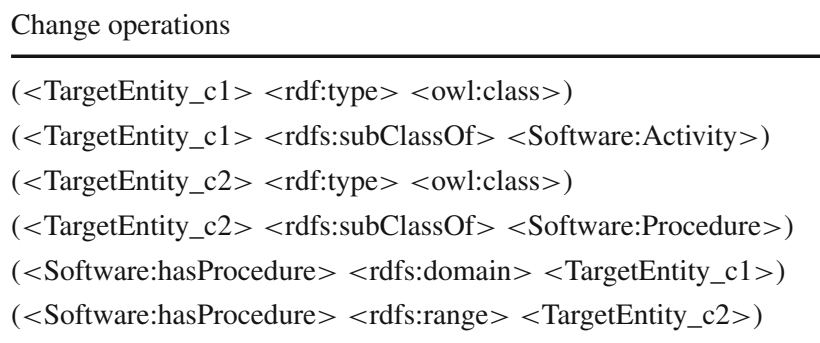

The example in Table 6 is the ABox-based change pattern from the university ontology, representing the registration procedure of a new $\mathrm{PhD}$ student to the department. First, the student has been registered as a $\mathrm{PhD}$ student of a particular department. Then, a student id, email id and a supervisor (which is a faculty member of the university) are assigned to the student. At the end, the student is added as a member of a particular research group of the university. We captured such change patterns and stored them in the ontology evolution framework for their reuse. Hence, whenever a new PhD student has to be registered, a stored change pattern can be applied as a single transaction (ensuring cross-ontology integrity constraints to be met).

The example in Table 7 is a TBox-based change pattern from a software application ontology, representing the introduction of a new software activity. First, a new concept (TargetEntity_cl) has been added as a subclass of concept Software:Activity. Later, to perform this activity, a new procedure has been added as a subclass of concept Software:Procedure in the help infrastructure section of the ontology. Finally, the activity and the procedure to perform such an activity are linked to each other using an object property Software:hasProcedure.

\subsubsection{Evaluation}

We conducted a performance study on our case study datasets (Table 8). We utilized our algorithms to discover 
Table 8 Comparison b/w OCP and UCP Algorithm with Minimum Pattern Support (min_sup) $=5$ and Minimum Pattern Length $($ min_len $)=5$

\begin{tabular}{llllll}
\hline Node dist. & \multicolumn{2}{l}{ a: OCP Algorithm } & & \multicolumn{2}{l}{ b: UCP Algorithm } \\
\cline { 2 - 3 } & $\begin{array}{l}\text { Patterns } \\
\text { found }\end{array}$ & $\begin{array}{l}\text { Time } \\
(\mathrm{ms})\end{array}$ & & $\begin{array}{l}\text { Patterns } \\
\text { found }\end{array}$ & $\begin{array}{l}\text { Time } \\
(\mathrm{ms})\end{array}$ \\
\hline 0 & 0 & 469 & & 4 & 1359 \\
1 & 3 & 609 & & 7 & 2282 \\
2 & 5 & 8756 & & 6 & 3906 \\
3 & 5 & 985 & & 8 & 4968 \\
4 & 5 & 1110 & & 8 & 6078 \\
5 & 5 & 1203 & & 9 & 7141 \\
\hline
\end{tabular}

the domain-specific change patterns in ontology change log graphs. Given a fixed user input value for minimum pattern length and minimum pattern support, we executed the algorithms, varied the node-distance value and evaluated their results.

OCP is efficient in terms of time consumption due to the permissibility of only positive node distances $(x)$, i.e. the iteration process for the search of the next adjacent sequence node only operates in forward direction of the change log graph. However, in the case of UCP, for the search of the next adjacent sequence node, the algorithm also operates in backward direction. This is due to the possibility of change operations in an unordered form compared to the referenced candidate change sequence. Another reason for the efficiency of OCP is the immediate termination of node search iterations once the next adjacent sequence node is not identified in the search space. However, in case of UCP, if the next adjacent node is not identified, it is saved in the unidentified node list and the iteration moves forward to search for the next adjacent node until the whole change sequence ends. Unordered change operations make the UCP algorithm more complex in comparison to OCP as UCP needs to (1) keep record of all change operations of the sequence (even if they are not identified), (2) recalculate the search space in each iteration, (3) search the next sequence node not only in the search space of the graph but also in the unidentified list of change nodes and (4) converting a sequence to ascending form in each iteration. UCP is more efficient in terms of numbers of discovered patterns. It discovers more change patterns compared to OCP (9:5). Similarly, in terms of the size of maximal patterns, UCP discovers patterns of greater size than OCP.

\subsubsection{Analysis of Discovered Change Patterns}

In this section, we examine the practical benefits of the discovered change patterns and lessons learnt in existing real world scenario. Possible applications of our pattern discovery algorithms range from supporting the change tracking tools, identification of user's behavioural dependency and classification of users [12], change request recommendations, analysis of change patterns and discovery of causal dependencies.

1. Tool Support for Change Tracking:One of the key benefits of our change patterns discovery approach is its integration with an existing ontology change tracking toolkit (such as Protégé, Neon, etc.). Users can choose a suitable change patterns from the discovered change pattern list and store them in their user profile. Later, whenever users load that particular ontology, they get the list of stored change patterns in their profile and can apply these in the form of transactions.

2. Change Request Recommendation: The identified change patterns can also be used for change request recommendations. For example, whenever a user adds a new $\mathrm{PhD}$ student in the university ontology, based on the identified $P h D$ Student Registration change pattern, it can be recommended to the user to add student id, email id of the student and assign a supervisor to him (using object property hasSupervisor). Similarly in software application domain, whenever a user deletes a certain activity from the domain, the deletion of the relevant help files can also be recommended to the user.

A limitation of our algorithms is that they cannot be applied on the change parameters which are represented as a complex expressions. Our algorithm considers all parameters as atomic classes, properties or individuals based on the OWL 2 working draft specification.

\section{Conclusion}

In this paper, we discussed our approach for ontology evolution as a pattern-based compositional framework. The approach focuses on a four-phase ontology change management system that performs and records changes at a higher level of granularity. We presented a layered change log model that works in line with the given layered change operator framework. While ontology engineers typically deal with generic changes at level one and level two, other users (such as domain experts, content managers) can focus on domainspecific changes at level three. Such a layered change operator framework enables us to deal with structural and semantic changes at two separate levels without losing their interdependence. Plus, it enables us to define a set of domain-specific changes which can be stored in a pattern catalogue, using a pattern template, as a consistent once-off specification of domain-specific change patterns. The empirical study indicates that the solution is valid and adequate to efficiently handle ontology evolution. We found that a significant portion of ontology change and evolution is represented in our framework. 
Identification of higher-level change operations gives an ontology engineer clues about semantics / reasons behind any of the applied change, based on the actual change activity data from change log. We operationalized the identification of higher-level changes using graph-based matching and pattern discovery approaches. We noticed that learning about semantics behind any of the applied change helped us in keeping the ontology consistent in a more appropriate manner. To do so, higher level evolutionary strategies are essential.

Constructing and storing the domain knowledge using a frame-based approach were introduced in the ProtégéFrames editor. It allows a user to construct customizable domain-specific data entry forms and entering the instancelevel data. As the concept hierarchy as well as the description about any concept will evolve through time, such dataentry forms will get obsolete unless customized through time. Discovery of the domain-specific change patterns from the change log can assist in this regard. It not only allows defining new "usage-driven" domain-specific change patterns, but can also aid in customization and editing of already available "user-defined" data entry forms. As good patterns always arise from practical experience [42], such change patterns, created in a collaborative environment, provide guidelines to ontology change management and can be used in any change recommendation system.

More research work needs to be done to address the limitations regarding the reusability of the (defined) change patterns. A highly reused change pattern indicates that it is generally accepted within the domain. The reusability of the discovered domain-specific change patterns can be enhanced through domain transfer. During our empirical study, we observed similarities of patterns across domains which are similar to each other. For example, in the university domain, one can identify classes such as students, faculties and employees; a production company may have employees, customers, owners or shareholders. The change patterns provided at higher level can be applied to any subject domain ontology that is composed of a similar conceptual structure. The domain-specific change patterns may require a small customization to meet the domain's own requirements. Similarity between two domain ontologies can be acknowledged by analyzing conceptual and syntactical structures within the domain ontologies.

Good documentation is vital for effective reuse of any framework. To address the limitations regarding documentation, our future work includes a specification of the (userdefined/usage-driven) domain-specific change patterns to support the notion of pattern-based ontology evolution. More specifically, we are interested in the once-off specification of the domain-specific change patterns that assist the ontology engineer to choose the appropriate change pattern in a given ontology evolution context. This can be achieved by utiliz- ing a pattern template that enables a consistent change pattern specification for change patterns comprising descriptive and change data information.

Acknowledgments This research is supported by Science Foundation Ireland (Grant 07/CE/I1142) as part of the Centre for Next Generation Localisation at Dublin City University. The authors also acknowledge the insightful comments given by the reviewers, which have greatly helped to improve the paper.

\section{References}

1. Agrawal R, Gunopulos D, Leymann F (1998) Mining process models from workflow logs

2. Agrawal R, Srikant R (1995) Mining sequential patterns. In: Proceedings of the international conference on data engineering. IEEE Computer Society, New York, pp 3-14

3. Altschul S, Gish W, Miller W, Myers E, Lipman D (1990) Basic local alignment search tool. J Mol Biol 215(3):403-410

4. Auer S, Herre H (2007) A versioning and evolution framework for rdf knowledge bases. In: Proceedings of the 6th international Andrei Ershov memorial conference on Perspectives of systems informatics, PSI'06. Springer, Berlin, pp 55-69

5. Cook J, Wolf A (1998) Discovering models of software prosses from event-based data. ACM Trans Softw Eng Methodol 5(3):215249

6. Corradini A, Montanari U, Rossi F, Ehrig H, Heckel R, Loew M (1996) Algebraic approaches to graph transformation, part-I: Basic concepts and double pushout approach. In: Technical Report Tr96-17, Universita di Pisa, Dipartimento di, Informatica

7. Djedidi R, Aufaure MA (2010) Onto-evoal an ontology evolution approach guided by pattern modeling and quality evaluation. In: Proceedings of the 6th international conference on Foundations of Information and Knowledge Systems, FoIKS'10. Springer, Berlin, pp 286-305

8. Dongen BFV, van der Aalst WMP (2004) Multi-phase process mining: Building instance graphs. In: International conference on conceptual modeling (ER 2004). LNCS, vol 3288. Springer, Berlin, pp 362-376

9. Easterbrook S, Singer J, Storey MA, Damian D (2008) Selecting empirical methods for software engineering research. In: Guide to Advanced Empirical Software Engineering. Springer, Berlin, pp 285-311

10. Ehrig H, Pfender M, Schneider H (1973) Graph grammars: an algebraic approach. In: Proceedings of 14th annual IEEE symposium on switching and automata theory, pp 167-180

11. Ehrig H, Prange U, Taentzer G (2004) Fundamental theory for typed attributed graph transformation. In: International conference on graph transformation, pp 161-177

12. Falconer S, Tudorache T, Noy NF (2011) An analysis of collaborative patterns in large-scale ontology development projects. In: Proceedings of the sixth international conference on knowledge capture, K-CAP '11, pp 25-32

13. Flouris G, Manakanatas D, Kondylakis H, Plexousakis D, Antoniou G (2008) Ontology change: classification and survey. Camb J Knowl Eng Rev 23(02):117-152

14. Groner G, Staab S (2010) Categorization and recognition of ontology refactoring pattern. Technical Report 09/2010, Institut WeST, University of Koblenz-Landau

15. Gruhn V, Pahl C, Wever M (1995) Data model evolution as basis of business process management. In: Proceedings of the 14th international conference on object-oriented and entity-relationship modelling (OOER '95). Springer, Berlin, pp 270-281 
16. Haase P, Sure Y (2004) User-driven ontology evolution management. State-of-the-Art on Ontology Evolution. EU IST Project SEKT Deliverable D3 1.1.b

17. Hartung M, Gross A, Rahm E (2012) Conto-diff: generation of complex evolution mappings for life science ontologies. J Biomed Inform

18. Heckel R, Kuster JM, Taentzer G (2002) Confluence of typed attributed graph transformation systems. In: Proceedings of ICGT 2002. LNCS, vol 2505. Springer, Berlin, pp 161-176 (2002)

19. Henninger S, Ashokkumar P (2006) An ontology-based metamodel for software patterns. In: 18th international conference on software engineering and knowledge engineering (Seke), pp 327-330

20. Huan J (2006) Graph based pattern discovery in protein structures. $\mathrm{PhD}$ thesis, Department of Computer Science, University of North Carolina

21. Inokuchi A, Washio T, Motoda H (2000) An apriori-based algorithm for mining frequent substructures from graph data. In: Proceedings of the 4th European conference on principles of data mining and knowledge discovery, pp 13-23

22. Javed M, Abgaz YM, Pahl C (2009) A pattern-based framework of change operators for ontology evolution. In: On the move to meaningful internet systems: OTM workshops. LNCS, vol 5872. Springer, Berlin, pp 544-553

23. Javed M, Abgaz YM, Pahl C (2011) Graph-based discovery of ontology change patterns. In: ISWC workshops: joint workshop on knowledge evolution and ontology dynamics (EvoDyn), 24th October, Bonn, Germany

24. Javed M, Abgaz YM, Pahl C (2011) A layered framework for pattern-based ontology evolution. In: 3rd international workshop ontology-driven information system engineering (ODISE), London, UK

25. Javed M, Abgaz YM, Pahl C (2012) Composite ontology change operators and their customizable evolution strategies. In: ISWC workshops: joint workshop on knowledge evolution and ontology dynamics (EvoDyn), 12th November, Boston, USA

26. Kampffmeyer H, Zschaler S (2007) Finding the pattern you need: the design pattern intent ontology. In: Model driven engineering languages and systems (MODELS), pp 211-225

27. Klein M (2004) Change management for distributed ontologies. $\mathrm{PhD}$ thesis, Vrije University Amsterdam

28. Kondylakis H, Plexousakis D (2012) Ontology evolution: assisting query migration. In: Atzeni P, Cheung D, Sudha R (eds) LNCS, vol 7532. Springer, Berlin, pp 331-344

29. Konstantinidis G, Flouris G, Antoniou G, Christophides V (2008) A formal approach for rdf/s ontology evolution. In: Proceedings of the 2008 conference on ECAI 2008: 18th European conference on artificial intelligence. IOS Press, Fairfax, pp 70-74

30. Kuramochi M, Karypis G (2001) Frequent subgraph discovery. In: 1st IEEE conference on data mining, pp 313-320

31. Li C, Wang J (2008) Efficiently mining closed subsequences with gap constraints. In: Proceedings of SIAM international conference on data mining (SDM'08), USA, pp 313-322

32. Liang Y, Alani H, Shadbolt N (2005) Ontology change management in protégé. In: Proceedings of AKT DTA colloquium, Milton Keynes, UK

33. Noy NF, Klein M (2004) Ontology evolution: not the same as schema evolution. J Knowl Inf Syst 6(4):328-440
34. Palma R, Haase P, Corcho O, Gomez-Perez A (2009) Change representation for owl 2 ontologies. In: Proceedings of the sixth international workshop on OWL: experiences and directions (OWLED)

35. Papavassiliou V, Flouris G, Fundulaki I, Kotzinos D, Christophides V (2009) On detecting high-level changes in RDF/S KBs. In: 8th International semantic web conference. LNCS, vol 5823. Springer, Berlin, pp 473-488

36. Pedrinaci C, Domingue J (2007) Towards an ontology for process monitoring and mining. In: Proceedings of the workshop on semantic business process and product lifecycle management

37. Plantevit M, Laurent A, Laurent D, Teisseire M, Choong YW (2010) Mining multidimensional and multilevel sequential patterns. ACM Translations on Knowledge Discovery from Data, Article 4, vol 4(1)

38. Plessers P, De Troyer O (2005) Ontology change detection using a version log. In: 4th International semantic web conference. Springer, pp 578-592

39. Plessers P, De Troyer O, Casteleyn S (2007) Understanding ontology evolution: a change detection approach. Web Semant Sci Serv Agents World Wide Web 5(1):39-49

40. Qin L, Atluri V (2009) Evaluating the validity of data instances against ontology evolution over the semantic web. Inf Softw Technol 51(1):83-97

41. Rieß C, Heino N, Tramp S, Auer S (2010) Evopat-pattern-based evolution and refactoring of rdf knowledge bases. In: Proceedings of the 9th international semantic web conference on the semantic web. vol Part I, ISWC'10. Springer, Berlin, pp 647-662

42. Schmidt D, Fayad M, Johnson R (1996) Software patterns. In: Commun ACM Special Issue Patterns Pattern Lang 39(10):37-39

43. Stefanowski J (2007) Algorithms for context based sequential pattern mining. Fundamenta Informaticae 76(4):495-510

44. Stojanovic L (2004) Methods and tools for ontology evolution. $\mathrm{PhD}$ thesis, University of Karlsruhe

45. Stojanovic L, Maedche A, Motik B, Stojanovic N (2002) Userdriven ontology evolution management. In: Proceedings of the 13th international conference on knowledge engineering and knowledge management. Ontologies and the semantic Web, EKAW '02. Springer, Berlin, pp 285-300

46. Stojanovic L, Maedche A, Stojanovic N, Studer R (2003) Ontology evolution as reconfiguration-design problem solving. In: Proceedings of the 2nd international conference on knowledge capture

47. Weijters AJMM, van der Aalst WMP (2001) Process mining discovering workflow models from event-based data. In: Proceedings of the ECAI workshop on knowledge discovery and spatial data, pp 283-290

48. Wen L, Wang J, van der Aalst WMP, Huang B, Sun J (2010) Mining process models with prime invisible tasks. Data Knowl Eng 69(10):999-1021

49. Yan X, Han J (2002) gspan: Graph-based substructure pattern mining. In: IEEE international conference on data mining, pp 721-724

50. Zablith F (2008) Dynamic ontology evolution. In: International semantic web conference (ISWC) doctoral consortium, Karlsruhe, Germany

51. Zhu X, Wu X (2007) Mining complex patterns across sequences with gap requirements. In: 20th international joint conference on artificial intelligence, pp 2934-2940 